\title{
Variants of Kato's inequality and removable singularities
}

\author{
Juan Dávila and Augusto C. Ponce* \\ E-mails: davila@math.rutgers.edu, augponce@math.rutgers.edu \\ Department of Mathematics - Hill Center \\ Rutgers, The State University of New Jersey \\ 110 Frelinghuysen Rd. \\ Piscataway, NJ 08854-8019 USA
}

\begin{abstract}
An inequality reminiscent of Kato's inequality is presented. Motivated by this, we discuss some criteria to decide whether a singularity of the equation $\Delta u=g$ in $\Omega \backslash K$ comes from a Radon measure or not. As an application, we extend a lemma of H. Brezis and P.L. Lions on isolated singularities to the case where the singularity lies on a compact manifold.
\end{abstract}

\section{Introduction and main results}

The original motivation for this work is the following remark, which is related to Kato's inequality (see Kato $[\mathrm{K}]$ ). First, let us recall one of its many versions. Consider $\Omega \subset \mathbb{R}^{N}$ an open set, and $v \in L^{1}(\Omega)$ such that $\Delta v \in$ $L^{1}(\Omega)$. Then

$$
\Delta|v| \geq \operatorname{sign}(v) \Delta v \quad \text { in } \mathcal{D}^{\prime}(\Omega),
$$

where $\operatorname{sign}(s)=1$ if $s>0,-1$ if $s<0$ and zero at $s=0$. If we assume in addition that $v$ is continuous in $\Omega$, it is easy to verify that

$$
\Delta|v|=\operatorname{sign}(v) \Delta v \quad \text { in } \mathcal{D}^{\prime}([v \neq 0]) .
$$

Comparison between (1) and (2) suggests that the inequality in (1) should be a consequence of the fact that $|v|$ achieves its minimum on the set $[v=0]$, where one has $\Delta|v| \geq 0$ in a suitable sense.

Motivated by this fact, Y. Li posed the following question: suppose $u \in$ $L^{1}(\Omega)$ is such that $u \geq 0$ a.e. in $\Omega$ and $u \equiv 0$ on a compact set $K$ in some reasonable sense. Set $g=\Delta u$ in $\mathcal{D}^{\prime}(\Omega \backslash K)$ and assume that $g \in L^{1}(\Omega \backslash K)$

\footnotetext{
${ }^{*}$ This author was supported by CAPES, Brazil, under the grant BEX1187/99-6.
} 
(no conditions on $\Delta u$ are prescribed on the "zero set" $K$ ). Let $\tilde{g}$ be the extension of $g$ to $\Omega$ such that $\tilde{g} \equiv 0$ on $K$. Define

$$
\mu:=\Delta u-\tilde{g} \quad \text { in } \mathcal{D}^{\prime}(\Omega),
$$

so that $\operatorname{supp}(\mu) \subset K$. Is it true that $\mu$ is a nonnegative distribution? In this case it has to be a Radon measure supported in $K$ (see Schwartz [S]).

We have given a positive answer to this question in the following theorem, which includes the case where $u \in C(\Omega)$ and $K \subset[u=0]$.

Theorem 1 Let $\Omega \subset \mathbb{R}^{N}$ be a bounded open subset, and $u \in L^{1}(\Omega)$ such that $u \geq 0$ a.e. in $\Omega$. Let $K \subset \Omega$ be compact. Set

$$
g:=\Delta u \quad \text { in } \mathcal{D}^{\prime}(\Omega \backslash K) .
$$

Assume that $g \in L^{1}(\Omega \backslash K)$ and that

$$
\limsup _{r \downarrow 0} f_{x \in K} u=0 .
$$

Let $\tilde{g}$ be the extension of $g$ to $\Omega$ such that $\tilde{g} \equiv 0$ on $K$. Then $\Delta u \geq \tilde{g}$ in $\mathcal{D}^{\prime}(\Omega)$, in other words,

$$
\int_{\Omega} u \Delta \varphi \geq \int_{\Omega} \tilde{g} \varphi, \quad \forall \varphi \in C_{0}^{\infty}(\Omega), \varphi \geq 0 .
$$

As we have pointed out before, the theorem above implies the following

Corollary 2 Let $\Omega \subset \mathbb{R}^{N}$ be open, bounded, and $u \in C(\Omega)$ be a nonnegative function. Let $K \subset \Omega$ be a compact subset such that $u \equiv 0$ on $K$. Set

$$
g:=\Delta u \quad \text { in } \mathcal{D}^{\prime}(\Omega \backslash K),
$$

and assume that $g \in L^{1}(\Omega \backslash K)$. Let $\tilde{g}$ be the extension of $g$ to $\Omega$ such that $\tilde{g} \equiv 0$ on $K$. Then $\Delta u \geq \tilde{g}$ in $\mathcal{D}^{\prime}(\Omega)$, in other words,

$$
\int_{\Omega} u \Delta \varphi \geq \int_{\Omega} \tilde{g} \varphi, \quad \forall \varphi \in C_{0}^{\infty}(\Omega), \varphi \geq 0 .
$$

Remark 1 We will see later that if the set $K$ is sufficiently small and a certain growth condition for $u$ near $K$ is prescribed, then one really has the equality $\Delta u=\tilde{g}$ in $\mathcal{D}^{\prime}(\Omega)$ (see Corollary 7). This is not the general case, though, as one can see by very simple examples. For instance, if $u(x):=\frac{1}{2}\left|x_{N}\right|$ for $x \in \mathbb{R}^{N}$, then $\Delta u=d x^{\prime}$ in $\mathcal{D}^{\prime}\left(\mathbb{R}^{N}\right)$, where $d x^{\prime}$ denotes the $(N-1)$-dimensional Lebesgue measure on $\left[x_{N}=0\right]$. 
Remark 2 A consequence of this theorem is that $\mu=\Delta u-\tilde{g}$ is a nonnegative distribution, and hence a Radon measure. This implies that $u \in$ $W_{\text {loc }}^{1, p}(\Omega)$ for any $1 \leq p<N /(N-1)$ (see Bénilan-Brezis-Crandall $[\mathrm{BeBrC}]$ ).

Remark 3 The same theorem holds under the weaker hypothesis that $\Omega$ is just open, $K \subset \Omega$ is relatively closed, and

$$
\lim _{r \downarrow 0} \sup _{x \in A} f_{B_{r}(x)} u=0 \quad \text { for all } A \subset K \text { compact. }
$$

In fact, for any $\delta>0$ set

$$
\widehat{\Omega}_{\delta}:=\left\{x \in \Omega: d(x, \partial \Omega)>\delta \text { and }|x|<\frac{1}{\delta}\right\} .
$$

Now fix $\delta>0$ and let $\psi \in C_{0}^{\infty}\left(\widehat{\Omega}_{2 \delta}\right), 0 \leq \psi \leq 1$ and $\psi \equiv 1$ in $\widehat{\Omega}_{3 \delta}$. We can apply then Theorem 1 in $\widehat{\Omega}_{\delta}$ to $\hat{u}:=u \psi, \hat{K}:=K \cap \widehat{\Omega}_{2 \delta}$, and conclude that (4) holds for all $\varphi \in C_{0}^{\infty}\left(\widehat{\Omega}_{3 \delta}\right)$.

Remark 4 A simple application of the Besicovitch Covering Lemma implies that condition (3) is equivalent to

$$
\lim _{r \downarrow 0} \frac{1}{r^{N}} \int_{N_{r}(K)} u=0,
$$

where $N_{r}(K)$ denotes the $r$-neighborhood of $K$, i.e.,

$$
N_{r}(K)=\left\{x \in \mathbb{R}^{N} \mid \operatorname{dist}(x, K)<r\right\} .
$$

The assumption required in (3) (or equivalently (6)) is probably too strong but we do not know how to weaken it in this general setting. In the case where $K \subset \Omega$ is a smooth manifold of codimension 1, we have been able to relax the hypothesis (3) by assuming that

$$
\lim _{r \downarrow 0} f_{\Xi_{r}(K)} u=0
$$

where $\Xi_{r}=\Xi_{r}(K)$ is the tubular neighborhood of $K$ with radius $r$. In other words, for such singular sets, one can replace the factor $\frac{1}{r^{N}}$ in (6) by $\frac{1}{r}$, and still get the same conclusion of Theorem 1. More precisely, 
Theorem 3 Let $\Omega \subset \mathbb{R}^{N}$ be an open set and $M^{N-1} \subset \Omega$ be a compact, smooth manifold, without boundary, of codimension 1 . Let $u \in L_{\mathrm{loc}}^{1}(\Omega)$, and assume that there exists $g \in L_{\mathrm{loc}}^{1}(\Omega)$ such that

$$
\Delta u=g \quad \text { in } \mathcal{D}^{\prime}(\Omega \backslash M) .
$$

If

$$
\lim _{r \downarrow 0} \frac{1}{r} \int_{\Xi_{r}}|u|=0,
$$

then, for each $\varphi \in C_{0}^{\infty}(\Omega), \frac{1}{r^{2}} \int_{\Xi_{r}} u \varphi$ converges as $r \downarrow 0$, and

$$
\lim _{r \downarrow 0} \frac{1}{r^{2}} \int_{\Xi_{r}} u \varphi=\frac{1}{2} \int_{\Omega} u \Delta \varphi-g \varphi, \quad \forall \varphi \in C_{0}^{\infty}(\Omega) .
$$

In particular, if we suppose in addition that $u \geq 0$ a.e. in $\Omega$, then

$$
\Delta u \geq g \quad \text { in } \mathcal{D}^{\prime}(\Omega)
$$

Remark 5 As mentioned in Remark 2, a posteriori we conclude from (9) that $u \in W_{\text {loc }}^{1, p}(\Omega)$ for $1 \leq p<N /(N-1)$, in which case condition (7) is equivalent to $u=0$ in $M$ in the sense of the trace.

Next, we study the case where the singular set $M$ is a compact manifold of codimension $k \geq 2$. It turns out that, in this case, the condition $u \geq 0$ a.e. in $\Omega$ already suffices to conclude that $-\Delta u$ is a (nonnegative) measure on $M$. More precisely, we have

Theorem 4 Let $\Omega \subset \mathbb{R}^{N}$ be a bounded open set and let $M \subset \Omega$ be a compact, smooth manifold without boundary of codimension $k \geq 2$. Let $u \in L_{\mathrm{loc}}^{1}(\Omega), u \geq 0$ a.e. in $\Omega$, and assume there exists $g \in L_{\mathrm{loc}}^{1}(\Omega)$ such that

$$
\Delta u=g \quad \text { in } \mathcal{D}^{\prime}(\Omega \backslash M) .
$$

Set

$$
\mu:=\Delta u-g \quad \text { in } \mathcal{D}^{\prime}(\Omega),
$$

which is a distribution supported on $M$.

Then

$$
\mu \text { is a nonpositive measure on } M
$$


and, for any $\varphi \in C_{0}^{\infty}(\Omega)$, we have

$$
\langle\mu, \varphi\rangle= \begin{cases}-2(k-2) \lim _{r \downarrow 0} \frac{1}{r^{2}} \int_{\Xi_{r}} u \varphi & \text { if } k \geq 3, \\ -2 \lim _{r \downarrow 0} \frac{1}{r^{2}|\log r|} \int_{\Xi_{r}} u \varphi & \text { if } k=2 .\end{cases}
$$

We should mention that the conclusion (11) holds true in a much more general setting. In fact, a classical result in Potential Theory states that if in the statement above one replaces $M$ by a compact set of zero $H^{1}$-capacity $K$ (this includes the case of a smooth manifold of codimension $k \geq 2$ ) then $\mu$, defined by (10), is a nonpositive measure on $K$ (see L.L. Helms [H], Theorem 7.7). We present in Section 5 a completely independent proof of this result in our special case in order to deduce (12), which is used to prove Theorems 5 and 6 below.

Even if we do not assume any conditions on the sign of $u$, we can still characterize the case when $\mu$ is a measure in terms of the growth of $|u|$ near $M$. More precisely, we have proved the following

Theorem 5 Let $\Omega \subset \mathbb{R}^{N}$ be a bounded open set and let $M \subset \Omega$ be a compact, smooth manifold without boundary of codimension $k \geq 3$. Let $u \in L_{\text {loc }}^{1}(\Omega)$ (here we do not assume that $u \geq 0$ a.e. in $\Omega$ ) and assume there exists $g \in L_{\mathrm{loc}}^{1}(\Omega)$ such that

$$
\Delta u=g \quad \text { in } \mathcal{D}^{\prime}(\Omega \backslash M) .
$$

Set

$$
\mu:=\Delta u-g \quad \text { in } \mathcal{D}^{\prime}(\Omega),
$$

which is a distribution supported in $M$. Then $\mu$ is a Radon measure if and only if

$$
\frac{1}{r^{2}} \int_{\Xi_{r}}|u| \quad \text { remains bounded as } \quad r \downarrow 0 .
$$

In this case, for all $\varphi \in C_{0}^{\infty}(\Omega)$ we have

$$
\lim _{r \downarrow 0} \frac{1}{r^{2}} \int_{\Xi_{r}} u \varphi \quad \text { exists and equals }-\frac{1}{2(k-2)}\langle\mu, \varphi\rangle .
$$

Moreover,

$$
\lim _{r \downarrow 0} \frac{1}{r^{2}} \int_{\Xi_{r}}|u| \quad \text { exists and equals } \frac{1}{2(k-2)}\|\mu\|,
$$


where $\|\mu\|:=\sup \left\{\int_{M} w d \mu ; w \in C(M),\|w\|_{\infty} \leq 1\right\}$ denotes the usual norm of Radon measures on $M$.

Remark 6 Using a formula deduced in Section 3, we show (see Remark 9) that (14) still holds if one replaces (13) by

$$
\lim _{r \downarrow 0} \frac{1}{r} \int_{\Xi_{r}}|u|=0 .
$$

On the other hand, if one takes for instance the function $u(x)=\frac{x_{1}}{|x|^{3}}$ in $\mathbb{R}^{3} \backslash\{0\}$, then $\Delta u=c D_{x_{1}} \delta_{0}$ for some constant $c \neq 0$. In the notation of Theorem 5 , let $M:=\{0\}, g \equiv 0$ and $\mu:=c D_{x_{1}} \delta_{0}$, so that $\mu$ is a distribution of order 1 and

$$
\lim _{r \downarrow 0} \frac{1}{r} \int_{B_{r}}|u|>0 .
$$

The example above suggests the following

Open problem. Let $M \subset \Omega$ be a compact, smooth manifold without boundary of codimension $k \geq 3$. Let $u$ and $g$ be as in the statement of Theorem 5 , and set $\mu:=\Delta u-g$ in $\mathcal{D}^{\prime}(\Omega)$. If (16) holds, is $\mu$ a measure?

There is also a result analogous to Theorem 5 in the case of codimension $k=2$ :

Theorem 6 Let $\Omega \subset \mathbb{R}^{N}$ be a bounded open set and let $M \subset \Omega$ be a compact, smooth manifold without boundary of codimension $k=2$. Let $u \in L_{\text {loc }}^{1}(\Omega)$ (here we do not assume that $u \geq 0$ a.e. in $\Omega$ ) and assume there exists $g \in L_{\mathrm{loc}}^{1}(\Omega)$ such that

$$
\Delta u=g \quad \text { in } \mathcal{D}^{\prime}(\Omega \backslash M) .
$$

Set

$$
\mu:=\Delta u-g \quad \text { in } \mathcal{D}^{\prime}(\Omega),
$$

which is a distribution supported in $M$. Then $\mu$ is a Radon measure if and only if

$$
\frac{1}{r^{2}|\log r|} \int_{\Xi_{r}}|u| \quad \text { remains bounded as } \quad r \downarrow 0 \text {. }
$$

In this case, for all $\varphi \in C_{0}^{\infty}(\Omega)$ we have

$$
\lim _{r \downarrow 0} \frac{1}{r^{2}|\log r|} \int_{\Xi_{r}} u \varphi \quad \text { exists and equals } \quad-\frac{1}{2}\langle\mu, \varphi\rangle .
$$


Moreover,

$$
\lim _{r \downarrow 0} \frac{1}{r^{2}|\log r|} \int_{\Xi_{r}}|u| \quad \text { exists and equals } \quad \frac{1}{2}\|\mu\| .
$$

As a consequence of Theorems 5 and 6 we have the following removable singularity statement:

Corollary 7 (Removable singularity) Under the assumptions of Theorems 5 and 6 above, we have $\Delta u \in L_{\mathrm{loc}}^{1}(\Omega)$ if and only if

$$
\begin{array}{r}
\lim _{r \downarrow 0} \frac{1}{r^{2}} \int_{\Xi_{r}}|u|=0, \quad \text { for } k \geq 3, \\
\lim _{r \downarrow 0} \frac{1}{r^{2}|\log r|} \int_{\Xi_{r}}|u|=0, \quad \text { for } k=2 .
\end{array}
$$

Next, we give an application of Theorem 4, by extending an earlier result of Brezis-Lions $[\mathrm{BrL}]$ originally concerning the study of isolated singularities:

Theorem 8 Let $\Omega \subset \mathbb{R}^{N}$ be an open set and $M^{N-k} \subset \Omega$ be a compact manifold, without boundary, of codimension $k \geq 2$. Let $u \in L_{\mathrm{loc}}^{1}(\Omega \backslash M)$ be such that

$$
\begin{aligned}
& \Delta u \in L_{\mathrm{loc}}^{1}(\Omega \backslash M) \quad \text { in the sense of distributions on } \Omega \backslash M, \\
& u \geq 0 \text { a.e. in } \Omega, \\
& \Delta u \leq a u+f \quad \text { a.e. in } \Omega \backslash M,
\end{aligned}
$$

where $a$ is a nonnegative constant and $f \in L_{\mathrm{loc}}^{1}(\Omega)$.

Then $u \in L_{\text {loc }}^{1}(\Omega)$, and there exist $h \in L_{\text {loc }}^{1}(\Omega)$ and a nonnegative Radon measure $\mu$ supported on $M$ such that

$$
-\Delta u=h+\mu \quad \text { in } \mathcal{D}^{\prime}(\Omega) .
$$

Since a compact manifold $M$ of codimension $k \geq 2$ is a set of zero $H^{1}$ capacity, and also because of the linear nature of Theorem 8 , the classical result we mention just after Theorem 4 leads us to state the following

Open problem. Suppose in the statement of Theorem 8 one replaces the smooth manifold $M$ by a compact set $K$ of zero $H^{1}$-capacity. Can one still conclude that $u \in L_{\mathrm{loc}}^{1}(\Omega)$, and that there exists $h \in L_{\mathrm{loc}}^{1}(\Omega)$ such that (23) 
holds for some $\mu$ supported on $K$ ? (Note that the Potential Theory would tell us that $\mu$ is necessarily a nonnegative Radon measure).

If the open problem above is true, it will give a sort of linear version of a general result of P. Baras and M. Pierre (see [BaPi]).

An immediate consequence of Theorem 8 is the following

Corollary 9 Let $M \subset \Omega$ be as above. Assume $f: \mathbb{R}_{+} \rightarrow \mathbb{R}$ is continuous and such that

$$
\liminf _{t \rightarrow \infty} \frac{f(t)}{t}>-\infty
$$

Suppose $u, f(u) \in L_{\mathrm{loc}}^{1}(\Omega \backslash M), u \geq 0$ a.e. in $\Omega$, and

$$
-\Delta u=f(u) \quad \text { in } \mathcal{D}^{\prime}(\Omega \backslash M) .
$$

Then $u, f(u) \in L_{\mathrm{loc}}^{1}(\Omega)$ and

$$
-\Delta u=f(u)+\mu \quad \text { in } \mathcal{D}^{\prime}(\Omega)
$$

for some nonnegative Radon measure $\mu$ supported on $M$.

A simple application of Corollaries 7 and 9 allows us to regain the following consequence of a removable singularity result which was originally proved by L. Véron for the case $k>2$ (see [V1]).

Corollary 10 Under the hypotheses of Corollary 9, if

$$
\begin{aligned}
& \liminf _{t \rightarrow \infty} t^{-\frac{k}{k-2}} f(t)>0, \quad \text { for } k>2, \\
& \liminf _{t \rightarrow \infty} \mathrm{e}^{-a t} f(t)>0, \quad \text { for } k=2, \text { for all } a>0,
\end{aligned}
$$

then $\mu=0$, i.e.,

$$
-\Delta u=f(u) \text { in } \mathcal{D}^{\prime}(\Omega) .
$$

Warning. The result of Corollary 10 may seem misleading at first. For instance, assume $k \geq 3$ and $f(t)=t^{\frac{k}{k-2}}$. Although it implies that $-\Delta u=$ $u^{\frac{k}{k-2}}$ in $\mathcal{D}^{\prime}(\Omega)$, one cannot conclude solely from this equation that $u$ is smooth. What Corollary 10 tells us is that the eventual singularities of $u$ are not detectable in the distribution level. In fact, a result of MazzeoPacard [MPa] says that, given some compact manifolds in $\Omega$ (not necessarily with the same codimension), and for certain values of $p>1$, depending on their codimension, one can construct nonnegative solutions of the equation $-\Delta u=u^{\frac{k}{k-2}}$ in $\mathcal{D}^{\prime}(\Omega)$, whose singularities lie precisely on the prescribed manifolds. See Véron [V2] for details. 


\section{Proof of Theorem 1}

In this section we shall use the following notation:

Notation. For an open set $U \subset \mathbb{R}^{N}$ and $\delta>0$ we write

$$
U_{\delta}=\{x \in U \mid d(x, \partial U)>\delta\},
$$

and for any set $A \subset \mathbb{R}^{N}$ and $\delta>0$ we let

$$
N_{\delta}(A)=\left\{x \in \mathbb{R}^{N} \mid d(x, A)<\delta\right\} .
$$

We also use the standard notation for averages:

$$
f_{E} v d \mu=\frac{\int_{E} v d \mu}{\int_{E} 1 d \mu}
$$

Proof of Theorem 1. Take $\rho \in C_{0}^{\infty}\left(B_{1}\right)$ such that $\rho \geq 0$ in $\mathbb{R}^{N}$ and $\int_{\mathbb{R}^{N}} \rho=1$. For any $\varepsilon>0$, define $\rho_{\varepsilon}(x):=\varepsilon^{-N} \rho(x / \varepsilon)$ on $\mathbb{R}^{N}, u_{\varepsilon}:=\rho_{\varepsilon} * u$ and $g_{\varepsilon}:=\rho_{\varepsilon} * \tilde{g}$ on $\Omega_{\varepsilon}$. Using this notation, one can easily check that

$$
\Delta u_{\varepsilon}=g_{\varepsilon} \quad \text { on } \Omega_{2 \varepsilon} \backslash \overline{N_{2 \varepsilon}(K)} .
$$

For $\varepsilon>0$, let

$$
\eta_{\varepsilon}:=\frac{\max }{N_{2 \varepsilon}(K)} u_{\varepsilon}
$$

Step 1. Condition (3) implies that

$$
\lim _{\varepsilon \downarrow 0} \eta_{\varepsilon}=0
$$

In particular, $u_{\varepsilon} \rightarrow 0$ uniformly on $K$ as $\varepsilon \downarrow 0$.

Proof. For $z \in \overline{N_{2 \varepsilon}(K)}$, let $x \in K$ be such that $|x-z| \leq 2 \varepsilon$. Since $B_{\varepsilon}(z) \subset B_{3 \varepsilon}(x)$ for all $\varepsilon>0$, we have

$$
\begin{aligned}
u_{\varepsilon}(z) & =\frac{1}{\varepsilon^{N}} \int_{B_{\varepsilon}(z)} \rho\left(\frac{z-y}{\varepsilon}\right) u(y) d y \\
& \leq \frac{C}{\varepsilon^{N}} \int_{B_{\varepsilon}(z)} u \leq \frac{C}{\varepsilon^{N}} \int_{B_{3 \varepsilon}(x)} u \\
& =\frac{3^{N} C}{(3 \varepsilon)^{N}} \int_{B_{3 \varepsilon(x)}} u \rightarrow 0
\end{aligned}
$$


uniformly in $z \in \overline{N_{2 \varepsilon}(K)}$ as $\varepsilon \rightarrow 0$, by (3). This concludes the proof of the claim.

Step 2. There exists a measurable set $L(u) \subset \Omega$ such that

$$
\left[\liminf u_{\varepsilon}>0\right] \subset L(u) \subset \Omega \backslash K
$$

and

$$
\int_{\Omega} u \Delta \varphi \geq \int_{L(u)} \tilde{g} \varphi, \quad \forall \varphi \in C_{0}^{\infty}(\Omega), \varphi \geq 0 .
$$

Proof. It follows from Kato's inequality (with $|\cdot|$ replaced by sign ${ }^{+}$in (1)) that

$$
\begin{aligned}
\Delta\left(u_{\varepsilon}-\eta_{\varepsilon}\right)^{+} & \geq \operatorname{sign}^{+}\left(u_{\varepsilon}-\eta_{\varepsilon}\right) \Delta\left(u_{\varepsilon}-\eta_{\varepsilon}\right) \\
& =\chi_{\left[u_{\varepsilon}>\eta_{\varepsilon}\right]} \Delta u_{\varepsilon} \quad \text { in } \mathcal{D}^{\prime}\left(\Omega_{2 \varepsilon}\right),
\end{aligned}
$$

where $\chi_{\left[u_{\varepsilon}>\eta_{\varepsilon}\right]}$ is the characteristic function of the set $\left[u_{\varepsilon}>\eta_{\varepsilon}\right]$.

Since $u_{\varepsilon} \leq \eta_{\varepsilon}$ on $\overline{N_{2 \varepsilon}(K)}$, it follows from (26) and (28) that

$$
\Delta\left(u_{\varepsilon}-\eta_{\varepsilon}\right)^{+} \geq \chi_{\left[u_{\varepsilon}>\eta_{\varepsilon}\right]} g_{\varepsilon} \quad \text { in } \mathcal{D}^{\prime}\left(\Omega_{2 \varepsilon}\right) .
$$

Now, given $\varphi \in C_{0}^{\infty}(\Omega)$ such that $\varphi \geq 0$, if $\varepsilon>0$ is sufficiently small so that $\operatorname{supp} \varphi \subset \subset \Omega_{2 \varepsilon},(29)$ implies that

$$
\int_{\Omega}\left(u_{\varepsilon}-\eta_{\varepsilon}\right)^{+} \Delta \varphi \geq \int_{\Omega} \chi_{\left[u_{\varepsilon}>\eta_{\varepsilon}\right]} g_{\varepsilon} \varphi .
$$

Since $u_{\varepsilon} \rightarrow u$ in $L_{\text {loc }}^{1}(\Omega)$ and $\eta_{\varepsilon} \rightarrow 0$ as $\varepsilon \rightarrow 0$, we conclude that

$$
\int_{\Omega}\left(u_{\varepsilon}-\eta_{\varepsilon}\right)^{+} \Delta \varphi \rightarrow \int_{\Omega} u \Delta \varphi \quad \text { as } \varepsilon \rightarrow 0
$$

On the other hand, take a sequence $\varepsilon_{n} \downarrow 0$. Up to a subsequence of $\left(\varepsilon_{n}\right)_{n \geq 1}$, we have

$$
\begin{array}{ll}
u_{\varepsilon_{n}} \rightarrow u & \text { a.e. in } \Omega, \\
g_{\varepsilon_{n}} \rightarrow \tilde{g} & \text { a.e. in } \Omega, \\
\left|g_{\varepsilon_{n}}\right| \leq h & \text { a.e. in } \Omega, \forall n \geq 1, \text { for some } h \in L^{1}(\Omega)
\end{array}
$$

Set

$$
L(u):=\liminf _{n \rightarrow \infty}\left[u_{\varepsilon_{n}}>\eta_{\varepsilon_{n}}\right]=\bigcup_{k=1}^{\infty} \bigcap_{n=k}^{\infty}\left[u_{\varepsilon_{n}}>\eta_{\varepsilon_{n}}\right]
$$


Note that, by our choice of $\eta_{\varepsilon}$, we have $K \subset \Omega \backslash L(u)$. By Fatou's Lemma, which may be applied here since $g_{\varepsilon_{n}} \geq-h$ a.e. in $\Omega$, we have

$$
\int_{\Omega} \chi_{L(u)} \tilde{g} \varphi=\int_{\Omega} \liminf _{n \rightarrow \infty} \chi_{\left[u_{\varepsilon_{n}}>\eta_{\varepsilon_{n}}\right]} g_{\varepsilon_{n}} \varphi \leq \liminf _{n \rightarrow \infty} \int_{\Omega} \chi_{\left[u_{\varepsilon_{n}}>\eta_{\varepsilon_{n}}\right]} g_{\varepsilon_{n}} \varphi .
$$

It then follows from (30), (31) and (32) that

$$
\int_{\Omega} u \Delta \varphi \geq \int_{\Omega} \chi_{L(u)} \tilde{g} \varphi, \quad \forall \varphi \in C_{0}^{\infty}(\Omega), \varphi \geq 0 .
$$

\section{Step 3. Proof of Theorem 1 completed.}

Given $\lambda>0$, let $h_{\lambda} \in C^{\infty}\left(\mathbb{R}^{N}\right)$ be such that $h_{\lambda} \equiv 0$ on $\overline{N_{\lambda}(K)}$ and $h_{\lambda}>0$ outside $\overline{N_{\lambda}(K)}$.

If we apply (27) in Step 2 with the function $u$ replaced by $u+h_{\lambda}$ (note that condition (3) is still satisfied if we replace $u$ by $u+h_{\lambda}$ ), we get:

$$
\int_{\Omega}\left(u+h_{\lambda}\right) \Delta \varphi \geq \int_{\Omega} \chi_{L\left(u+h_{\lambda}\right)}\left(\tilde{g}+\Delta h_{\lambda}\right) \varphi, \quad \forall \varphi \in C_{0}^{\infty}(\Omega), \varphi \geq 0 .
$$

Now, for a.e. $x \in \Omega \backslash \overline{N_{\lambda}(K)}$ we have $\left(u+h_{\lambda}\right)_{\varepsilon_{n}}(x) \rightarrow u(x)+h_{\lambda}(x)>0$ as $n \rightarrow \infty$. By the definition of the set $L\left(u+h_{\lambda}\right)$, we conclude that $x \in L\left(u+h_{\lambda}\right)$ for a.e. $x \in \Omega \backslash \overline{N_{\lambda}(K)}$, in other words,

$$
\chi_{L\left(u+h_{\lambda}\right)}=1 \quad \text { a.e. in } \Omega \backslash \overline{N_{\lambda}(K)} .
$$

In view of (33) and the relation above, for any $\varphi \in C_{0}^{\infty}(\Omega)$,

$$
\begin{aligned}
\int_{\Omega} u \Delta \varphi & \geq \int_{\Omega} \chi_{L\left(u+h_{\lambda}\right)}\left(\tilde{g}+\Delta h_{\lambda}\right) \varphi-\int_{\Omega} \Delta h_{\lambda} \varphi \\
& =\int_{\Omega \backslash \overline{N_{\lambda}(K)}}\left(\tilde{g}+\Delta h_{\lambda}\right) \varphi+\int_{\overline{N_{\lambda}(K)}} \chi_{L\left(u+h_{\lambda}\right)} \tilde{g} \varphi-\int_{\Omega \backslash \overline{N_{\lambda}(K)}} \Delta h_{\lambda} \varphi \\
& =\int_{\Omega \backslash \overline{N_{\lambda}(K)}} \tilde{g} \varphi+\int_{\overline{N_{\lambda}(K)}} \chi_{L\left(u+h_{\lambda}\right)} \tilde{g} \varphi \\
& =\int_{\Omega} \tilde{g} \varphi+o(1),
\end{aligned}
$$

where $o(1)$ is a quantity which converges to 0 as $\lambda \downarrow 0$. In the expression above, let $\lambda \downarrow 0$ to finally conclude that

$$
\int_{\Omega} u \Delta \varphi \geq \int_{\Omega} \tilde{g} \varphi, \quad \forall \varphi \in C_{0}^{\infty}(\Omega), \varphi \geq 0 .
$$


Remark 7 It is worth noting that the proof of Theorem 1 is somewhat simpler if one assumes that $u$ is continuous at each point of $K$. In fact, in this case Step 1 would be unnecessary and one can apply the other steps of the proof directly to the function $u$ instead of to its convolution.

\section{Some useful formulas}

Let us recall some standard results.

Given a compact smooth manifold $M^{N-k}$ (with or without boundary) embedded in $\mathbb{R}^{N}$ with codimension $k \geq 1$, we define its distance function $d: \mathbb{R}^{N} \rightarrow \mathbb{R}_{+}$by $d(x):=\operatorname{dist}(x, M)$. The case $k=N$ is included, i.e., $M$ may be a finite collection of points. It is a well-known fact that for $\delta>0$ small enough, the set $\overline{N_{\delta}(M)}$ is a smooth manifold with boundary, also called the $\delta$-tubular neighborhood of $M$, which from now on we shall denote by $\Xi_{\delta}(M)$, and when no confusion arises, simply by $\Xi_{\delta}$. The distance function $d$ is Lipschitz in $\mathbb{R}^{N}$, it is smooth in $\Xi_{\delta} \backslash M$ and satisfies (for the second property see Véron [V2]):

$$
\begin{aligned}
|\nabla d| & =1 \quad \text { a.e. in } \mathbb{R}^{N} ; \\
\Delta d & =\frac{k-1}{d}+a_{0} \quad \text { in } \Xi_{\delta} \backslash M,
\end{aligned}
$$

where $a_{0}$ is a bounded function in $\Xi_{\delta} \backslash M$.

For each $x \in \Xi_{\delta}$, there exists a unique element $\pi(x) \in M$ for which the distance function is realized, i.e., such that $|x-\pi(x)|=d(x)$. The projection $\pi: \Xi_{\delta} \rightarrow M$ thus defined is also smooth.

For simplicity, from now on we shall assume that $\Xi_{2}$ is a smooth tubular neighborhood of $M$.

Finally, let us recall that if $v \in L^{1}\left(\mathbb{R}^{N}\right)$ we have by the coarea formula (see Evans and Gariepy [EG])

$$
\int_{\Xi_{\delta}} v=\int_{0}^{\delta} \int_{\partial \Xi_{r}} v d \sigma d r .
$$

Lemma 11 Let $\Omega \subset \mathbb{R}^{N}$ be a bounded open set and let $M \subset \Omega$ be a compact, smooth manifold without boundary of codimension $k \geq 1$.

Let $u \in L_{\mathrm{loc}}^{1}(\Omega)$, and assume there exists $g \in L_{\mathrm{loc}}^{1}(\Omega)$ such that

$$
\Delta u=g \quad \text { in } \mathcal{D}^{\prime}(\Omega \backslash M) \text {. }
$$


Set

$$
\mu:=\Delta u-g \text { in } \mathcal{D}^{\prime}(\Omega)
$$

which is a distribution supported on $M$.

For $k \geq 1$ and $t, r>0$ define

$$
G_{k}(r, t)= \begin{cases}\frac{1}{k} & \text { if } 0<t<r \\ -\frac{k-1}{k} \frac{r^{k}}{t^{k}} & \text { if } 0<r<t .\end{cases}
$$

Then for any $R \in(0,1)$ fixed and $\varphi \in C_{0}^{\infty}(\Omega)$, all the limits below exist and: a) if $k \geq 3$ then

$$
\begin{aligned}
\frac{1}{2(k-2)}\langle\mu, \varphi\rangle= & \lim _{r \downarrow 0}\left\{-\frac{1}{r^{2}} \int_{\Xi_{r}} u \varphi+\right. \\
& \left.+\frac{1}{r^{2}} \int_{0}^{R} G_{k}(r, t)\left(\int_{\Xi_{t}} 2 u \nabla \varphi \cdot \nabla d+u \varphi a_{0}\right) d t\right\} ;
\end{aligned}
$$

b) if $k=2$ then

$$
\begin{aligned}
\frac{1}{2}\langle\mu, \varphi\rangle= & \lim _{r \downarrow 0}\left\{-\frac{1}{r^{2}|\log r|} \int_{\Xi_{r}} u \varphi+\right. \\
& \left.+\frac{1}{r^{2}|\log r|} \int_{0}^{R} G_{2}(r, t)\left(\int_{\Xi_{t}} 2 u \nabla \varphi \cdot \nabla d+u \varphi a_{0}\right) d t\right\} ;
\end{aligned}
$$

c) if $k=1$ then

$$
\begin{aligned}
-\frac{1}{2}\langle\mu, \varphi\rangle=\lim _{r \downarrow 0} & \left\{-\frac{1}{r^{2}} \int_{\Xi_{r}} u \varphi+\frac{1}{r}\left(\lim _{t \downarrow 0} \int_{\partial \Xi_{t}} u \varphi\right)\right. \\
+ & \left.\frac{1}{r^{2}} \int_{0}^{r}\left(\int_{\Xi_{t}} 2 u \nabla \varphi \cdot \nabla d+u \varphi a_{0}\right) d t\right\} .
\end{aligned}
$$

Proof. The idea of the proof is first to derive the following

Claim. For any $\varphi \in C_{0}^{\infty}(\Omega)$ the function $s \rightarrow \int_{\partial \Xi_{s}} u \varphi$ is $C^{1}$ on $(0,1)$ and

$$
\begin{aligned}
\langle\Delta u, \varphi\rangle= & \int_{\Xi_{s}} u \Delta \varphi-2 \int_{\partial \Xi_{s}} u \nabla \varphi \cdot \nabla d+s^{k-1} \frac{d}{d s}\left(\frac{1}{s^{k-1}} \int_{\partial \Xi_{s}} u \varphi\right)- \\
& -\int_{\partial \Xi_{s}} u \varphi a_{0} .
\end{aligned}
$$


Proof of (40). We first assume that $u$ is smooth.

Fix a smooth, nonincreasing function $\Phi: \mathbb{R} \rightarrow \mathbb{R}$ such that $\Phi(t)=0$ for $t \geq 1$ and $\Phi(t)=1$ for $t \leq 0$. For $\varepsilon>0$ set

$$
\Phi_{\varepsilon}(t)=\Phi\left(\frac{t-1}{\varepsilon}\right) .
$$

Now let $\varphi \in C_{0}^{\infty}(\Omega)$ and for $\varepsilon, s>0$ define

$$
\varphi_{s, \varepsilon}(x)= \begin{cases}\varphi(x) & \text { if } x \in \Xi_{s} \\ \varphi(x) \Phi_{\varepsilon}(d(x) / s) & \text { if } x \in \Xi_{s(1+\varepsilon)} \backslash \Xi_{s} \\ 0 & \text { if } x \in \Omega \backslash \Xi_{s(1+\varepsilon)} .\end{cases}
$$

Observe that $\varphi_{s, \varepsilon} \equiv \varphi$ in $\Xi_{s}$ and $\varphi_{s, \varepsilon} \equiv 0$ in $\Omega \backslash \Xi_{s(1+\varepsilon)}$. We now compute in $\Xi_{s(1+\varepsilon)} \backslash \Xi_{s}$, using (35):

$$
\begin{aligned}
\Delta \varphi_{s, \varepsilon}=\Delta & \varphi \Phi_{\varepsilon}(d / s)+\frac{2}{s} \nabla \varphi \cdot \nabla d \Phi_{\varepsilon}^{\prime}(d / s) \\
& +\frac{1}{s^{2}} \varphi\left\{\Phi_{\varepsilon}^{\prime \prime}(d / s)+\frac{s}{d} \Phi_{\varepsilon}^{\prime}(d / s)\left(k-1+a_{0} d\right)\right\} .
\end{aligned}
$$

Since $\varphi_{s, \varepsilon}$ is an admissible test function we obtain

$$
\left\langle\Delta u, \varphi_{s, \varepsilon}\right\rangle=\int_{\Omega} u \Delta \varphi \Phi_{\varepsilon}(d / s)+I_{1}+I_{2}+I_{3}+I_{4}
$$

where

$$
\begin{aligned}
& I_{1}=\frac{2}{s} \int_{\Omega} u \nabla \varphi \cdot \nabla d \Phi_{\varepsilon}^{\prime}(d / s) \\
& I_{2}=\frac{1}{s^{2}} \int_{\Omega} u \varphi \Phi_{\varepsilon}^{\prime \prime}(d / s) \\
& I_{3}=\frac{k-1}{s} \int_{\Omega} u \frac{\varphi}{d} \Phi_{\varepsilon}^{\prime}(d / s) \\
& I_{4}=\frac{1}{s} \int_{\Omega} u \varphi a_{0} \Phi_{\varepsilon}^{\prime}(d / s) .
\end{aligned}
$$

Next we find the limit as $\varepsilon \downarrow 0$ of the four previous integrals. For this purpose we compute

$$
I_{1}=\frac{2}{\varepsilon s} \int_{\Xi_{s(1+\varepsilon)} \backslash \Xi_{s}} \Phi^{\prime}\left(\frac{d / s-1}{\varepsilon}\right) u \nabla \varphi \cdot \nabla d
$$


and by the coarea formula

$$
\begin{aligned}
& =\frac{2}{\varepsilon s} \int_{s}^{s(1+\varepsilon)}\left\{\Phi^{\prime}\left(\frac{r / s-1}{s}\right) \int_{\partial \Xi_{r}} u \nabla \varphi \cdot \nabla d\right\} d r \\
& =2 \int_{0}^{1}\left\{\Phi^{\prime}(t) \int_{\partial \Xi_{(1+\varepsilon t) s}} u \nabla \varphi \cdot \nabla d\right\} d t .
\end{aligned}
$$

We now let $\varepsilon \downarrow 0$ :

$$
\begin{aligned}
\lim _{\varepsilon \downarrow 0} I_{1} & =2 \int_{0}^{1} \Phi^{\prime}(t)\left\{\int_{\partial \Xi_{s}} u \nabla \varphi \cdot \nabla d\right\} d t \\
& =-2 \int_{\partial \Xi_{s}} u \nabla \varphi \cdot \nabla d .
\end{aligned}
$$

We now proceed with $I_{2}$ :

$$
\begin{aligned}
I_{2} & =\frac{1}{s^{2}} \int_{\Omega} u \varphi \Phi_{\varepsilon}^{\prime \prime}(d / s) \\
& =\frac{1}{\varepsilon^{2} s^{2}} \int_{\Xi_{s(1+\varepsilon)} \backslash \Xi_{s}} u \varphi \Phi^{\prime \prime}\left(\frac{d / s-1}{\varepsilon}\right) \\
& =\frac{1}{\varepsilon s} \int_{0}^{1}\left\{\Phi^{\prime \prime}(t) \int_{\partial \Xi_{s(1+\varepsilon t)}} u \varphi\right\} d t
\end{aligned}
$$

and integrating by parts

$$
I_{2}=\left[\frac{1}{\varepsilon s} \Phi^{\prime}(t) \int_{\partial \Xi_{s(1+\varepsilon t)}} u \varphi\right]_{t=0}^{t=1}-\int_{0}^{1}\left(\Phi^{\prime}(t)\left[\frac{d}{d \lambda} \int_{\partial \Xi_{\lambda}} u \varphi\right]_{\lambda=s(1+\varepsilon t)}\right) d t .
$$

Letting $\varepsilon \downarrow 0$ we arrive at

$$
\lim _{\varepsilon \downarrow 0} I_{2}=\frac{d}{d s} \int_{\partial \Xi_{s}} u \varphi .
$$

The computations for $I_{3}, I_{4}$ are similar and they yield

$$
\begin{aligned}
& \lim _{\varepsilon \downarrow 0} I_{3}=-\frac{k-1}{s} \int_{\partial \Xi_{s}} u \varphi \\
& \lim _{\varepsilon \downarrow 0} I_{4}=-\int_{\partial \Xi_{s}} u \varphi a_{0} .
\end{aligned}
$$


Thus, passing to the limit as $\varepsilon \downarrow 0$ in (41) and using (42)-(45) we get

$$
\begin{aligned}
\langle\Delta u, \varphi\rangle= & \int_{\Xi_{s}} u \Delta \varphi-2 \int_{\partial \Xi_{s}} u \nabla \varphi \cdot \nabla d+\frac{d}{d s} \int_{\partial \Xi_{s}} u \varphi-\frac{k-1}{s} \int_{\partial \Xi_{s}} u \varphi- \\
& -\int_{\partial \Xi_{s}} u \varphi a_{0} .
\end{aligned}
$$

But

$$
\frac{d}{d s} \int_{\partial \Xi_{s}} u \varphi-\frac{k-1}{s} \int_{\partial \Xi_{s}} u \varphi=s^{k-1} \frac{d}{d s}\left(\frac{1}{s^{k-1}} \int_{\partial \Xi_{s}} u \varphi\right),
$$

and therefore, combining (46) with (47) we find (40).

We now consider $u$ as in the statement of the lemma, i.e., $u \in L_{\text {loc }}^{1}(\Omega)$ so that $\mu:=\Delta u-g$ is a distribution with support in $M$, where $g \in L_{\text {loc }}^{1}(\Omega)$. Using a density argument, and the fact that $u \in W_{\text {loc }}^{1,1}(\Omega \backslash M)$, we deduce that the function $s \mapsto \int_{\partial \Xi_{s}} u \varphi$ is $C^{1}$ on $(0,1)$ and that

$$
\begin{aligned}
\langle\mu, \varphi\rangle= & \int_{\Xi_{s}}(u \Delta \varphi-g \varphi)-2 \int_{\partial \Xi_{s}} u \nabla \varphi \cdot \nabla d+s^{k-1} \frac{d}{d s}\left(\frac{1}{s^{k-1}} \int_{\partial \Xi_{s}} u \varphi\right)- \\
& -\int_{\partial \Xi_{s}} u \varphi a_{0} .
\end{aligned}
$$

At this point we distinguish the three cases: a) $k \geq 3$, b) $k=2$, and c) $k=1$.

a) Case: $k \geq 3$. Fix $R \in(0,1)$ and let $0<t<R$. Dividing (48) by $s^{k-1}$ and integrating over $s \in(t, R)$ we get

$$
\begin{aligned}
\frac{1}{(k-2) t^{k-2}}\langle\mu, \varphi\rangle=o(1) \frac{1}{t^{k-2}}-\frac{1}{t^{k-1}} \int_{\partial \Xi_{t}} u \varphi- \\
\quad-\int_{t}^{R}\left\{\frac{1}{s^{k-1}} \int_{\partial \Xi_{s}} u \varphi a_{0}+2 u \nabla \varphi \cdot \nabla d\right\} d s,
\end{aligned}
$$

where $o(1)$ denotes a quantity that goes to zero as $t \rightarrow 0$. Multiplying (49) by $t^{k-1}$ and integrating over $t \in(0, r)$ with $0<r<R$, we obtain

$$
\begin{aligned}
\frac{1}{2(k-2)}\langle\mu, \varphi\rangle=o(1) & -\frac{1}{r^{2}} \int_{\Xi_{r}} u \varphi- \\
& -\frac{1}{r^{2}} \int_{0}^{r} t^{k-1} \int_{t}^{R} \frac{1}{s^{k-1}} \int_{\partial \Xi_{s}} v d s d t,
\end{aligned}
$$


where we use the notation

$$
v=2 u \nabla \varphi \cdot \nabla d+u \varphi a_{0} .
$$

We now integrate by parts in the last term on the right hand side of (50):

$$
\begin{aligned}
\int_{t}^{R} \frac{1}{s^{k-1}}\left(\frac{d}{d s} \int_{\Xi_{s}} v\right) d s= & {\left[\frac{1}{s^{k-1}} \int_{\Xi_{s}} v\right]_{s=t}^{s=R}-\int_{t}^{R}(1-k) \frac{1}{s^{k}} \int_{\Xi_{s}} v d s } \\
= & \frac{1}{R^{k-1}} \int_{\Xi_{R}} v-\frac{1}{t^{k-1}} \int_{\Xi_{t}} v+ \\
& \quad+(k-1) \int_{t}^{R} \frac{1}{s^{k}} \int_{\Xi_{s}} v d s .
\end{aligned}
$$

Therefore

$$
\begin{aligned}
& \frac{1}{r^{2}} \int_{0}^{r} t^{k-1} \int_{t}^{R} \frac{1}{s^{k-1}} \int_{\partial \Xi_{s}} v d s d t=\frac{r^{k-2}}{k R^{k-1}} \int_{\Xi_{R}} v-\frac{1}{r^{2}} \int_{0}^{r} \int_{\Xi_{t}} v d t+ \\
& +\frac{k-1}{r^{2}} \int_{0}^{r} t^{k-1} \int_{t}^{R} \frac{1}{s^{k}} \int_{\Xi_{s}} v d s d t
\end{aligned}
$$

and changing the order of integration in the last term of (52) gives

$$
\int_{0}^{r} t^{k-1} \int_{t}^{R} \frac{1}{s^{k}} \int_{\Xi_{s}} v d s d t=\frac{1}{k} \int_{0}^{r} \int_{\Xi_{s}} v d s+\frac{r^{k}}{k} \int_{r}^{R} \frac{1}{s^{k}} \int_{\Xi_{s}} v d s .
$$

Then, (52) in combination with (53) yields

$$
\begin{array}{r}
\frac{1}{r^{2}} \int_{0}^{r} t^{k-1} \int_{t}^{R} \frac{1}{s^{k-1}} \int_{\partial \Xi_{s}} v d s d t=\frac{r^{k-2}}{k R^{k-1}} \int_{\Xi_{R}} v-\frac{1}{k r^{2}} \int_{0}^{r} \int_{\Xi_{t}} v d t+ \\
+\frac{k-1}{k} r^{k-2} \int_{r}^{R} \frac{1}{s^{k}} \int_{\Xi_{s}} v d s .
\end{array}
$$

Hence, using (54) in (50) we conclude that

$$
\begin{aligned}
\frac{1}{2(k-2)}\langle\mu, \varphi\rangle=o(1)-\frac{1}{r^{2}} \int_{\Xi_{r}} u \varphi+\frac{1}{k r^{2}} \int_{0}^{r} \int_{\Xi_{t}} v d t- \\
\quad-\frac{k-1}{k} r^{k-2} \int_{r}^{R} \frac{1}{s^{k}} \int_{\Xi_{s}} v d s \\
=o(1)-\frac{1}{r^{2}} \int_{\Xi_{r}} u \varphi+\frac{1}{r^{2}} \int_{0}^{R}\left(G_{k}(r, t) \int_{\Xi_{t}} v\right) d t,
\end{aligned}
$$


where $G_{k}$ is given by (36).

This establishes (37).

We now deal with

b) Case: $k=2$. Note that (48) is still valid, and since $k=2$ it takes the form

$$
\langle\mu, \varphi\rangle=o(1)-\int_{\partial \Xi_{s}} v+s \frac{d}{d s}\left(\frac{1}{s} \int_{\partial \Xi_{s}} u \varphi\right),
$$

where $v$ is given by (51). Dividing the last equation by $s$ and integrating over $s \in(t, R)$ we get

$$
(\log R-\log t)\langle\mu, \varphi\rangle=o(1)|\log t|-\int_{t}^{R} \frac{1}{s} \int_{\partial \Xi_{s}} v d s-\frac{1}{t} \int_{\partial \Xi_{t}} u \varphi .
$$

Multiplying by $t$ and integrating over $t \in(0, r)$ we obtain

$$
\frac{1}{2}\langle\mu, \varphi\rangle=o(1)-\frac{1}{r^{2}|\log r|} \int_{0}^{r} t \int_{t}^{R} \frac{1}{s} \int_{\partial \Xi_{s}} v d s d t-\frac{1}{r^{2}|\log r|} \int_{\Xi_{r}} u \varphi .
$$

But, integrating by parts

$$
\begin{aligned}
\int_{t}^{R} \frac{1}{s} \int_{\partial \Xi_{s}} v d s & =\left[\frac{1}{s} \int_{\Xi_{s}} v\right]_{s=t}^{s=R}+\int_{t}^{R} \frac{1}{s^{2}} \int_{\Xi_{s}} v d s \\
& =\frac{1}{R} \int_{\Xi_{R}} v-\frac{1}{t} \int_{\Xi_{t}} v+\int_{t}^{R} \frac{1}{s^{2}} \int_{\Xi_{s}} v d s .
\end{aligned}
$$

Hence, using Fubini we get

$$
\begin{aligned}
\int_{0}^{r} t \int_{t}^{R} \frac{1}{s} \int_{\partial \Xi_{s}} v d s & =\frac{r^{2}}{2 R} \int_{\Xi_{R}} v-\int_{0}^{r} \int_{\Xi_{t}} v d t+\int_{0}^{r} t \int_{t}^{R} \frac{1}{s^{2}} \int_{\Xi_{s}} v d s d t \\
& =\frac{r^{2}}{2 R} \int_{\Xi_{R}} v-\frac{1}{2} \int_{0}^{r} \int_{\Xi_{t}} v d t+\frac{r^{2}}{2} \int_{r}^{R} \frac{1}{s^{2}} \int_{\Xi_{s}} v d s .
\end{aligned}
$$

So, from (55) and (56) we infer that

$$
\begin{aligned}
\frac{1}{2}\langle\mu, \varphi\rangle=o(1)-\frac{1}{r^{2}|\log r|} \int_{\Xi_{r}} u \varphi+\frac{1}{2 r^{2}|\log r|} \int_{0}^{r} \int_{\Xi_{t}} v d t- \\
\quad-\frac{1}{2|\log r|} \int_{r}^{R} \frac{1}{t^{2}} \int_{\Xi_{t}} v d t \\
=o(1)-\frac{1}{r^{2}|\log r|} \int_{\Xi_{r}} u \varphi+\frac{1}{r^{2}|\log r|} \int_{0}^{R}\left(G_{2}(r, t) \int_{\Xi_{t}} v\right) d t,
\end{aligned}
$$


where $G_{2}$ is given by (36) with $k=2$.

This proves (38).

Finally

c) Case: $k=1$. This time (48) becomes

$$
\langle\mu, \varphi\rangle=\int_{\Omega} \Delta u \varphi-g \varphi-\int_{\partial \Xi_{s}} v+\frac{d}{d s} \int_{\partial \Xi_{s}} u \varphi .
$$

Integrate the previous relation over $s \in(t, \lambda)$ :

$$
(\lambda-t)\langle\mu, \varphi\rangle=o(1)-\int_{\Xi_{\lambda} \backslash \Xi_{t}} v+\int_{\partial \Xi_{\lambda}} u \varphi-\int_{\partial \Xi_{t}} u \varphi .
$$

where $o(1) \rightarrow 0$ as $\lambda \rightarrow 0$. Since $v=2 u \nabla \varphi \cdot \nabla d+u \varphi a_{0} \in L_{\text {loc }}^{1}(\Omega)$, letting $t \downarrow 0$ in (57) we see that $\lim _{t \downarrow} \int_{\partial \Xi_{t}} u \varphi$ exists and

$$
\lambda\langle\mu, \varphi\rangle=o(1)-\int_{\Xi_{\lambda}} v+\int_{\partial \Xi_{\lambda}} u \varphi-\left(\lim _{t \downarrow 0} \int_{\partial \Xi_{t}} u \varphi\right) .
$$

We now integrate (58) over $\lambda \in(0, r)$ and divide by $r^{2}$ to find

$$
\frac{1}{2}\langle\mu, \varphi\rangle=o(1)+\frac{1}{r^{2}} \int_{\Xi_{r}} u \varphi-\frac{1}{r}\left(\lim _{t \downarrow 0} \int_{\partial \Xi_{t}} u \varphi\right)-\frac{1}{r^{2}} \int_{0}^{r} \int_{\Xi_{t}} v d t
$$

which concludes the proof of the lemma.

\section{Proof of Theorem 3}

Set $\mu=\Delta u-g$. Suppose (7) holds. Then, since $\lim _{r \downarrow 0} \int_{\partial \Xi_{r}} u \varphi$ exists by Lemma 11, we conclude that

$$
\lim _{r \downarrow 0} \int_{\partial \Xi_{r}} u \varphi=0, \quad \forall \varphi \in C_{0}^{\infty}(\Omega) .
$$

On the other hand, given $\varepsilon>0$, (7) implies that there exists $\delta>0$ such that

$$
\int_{\partial \Xi_{r}}|u| \leq \varepsilon, \quad \forall r \in(0, \delta) .
$$


Therefore, we have

$$
\begin{aligned}
\left|\int_{0}^{r}\left(\int_{\Xi_{t}} 2 u \nabla \varphi \cdot \nabla d+u \varphi a_{0}\right) d t\right| & \leq C \int_{0}^{r}\left(\int_{\Xi_{t}}|u|\right) d t \\
& \leq C \int_{0}^{r} \varepsilon t d t=\varepsilon \frac{r^{2}}{2}, \quad \forall r \in(0, \delta) .
\end{aligned}
$$

Since $\varepsilon>0$ was arbitrary, we deduce that

$$
\lim _{r \downarrow 0} \frac{1}{r^{2}} \int_{0}^{r}\left(\int_{\Xi_{t}} 2 u \nabla \varphi \cdot \nabla d+u \varphi a_{0}\right) d t=0 .
$$

Inserting (59) and (60) into (39) we get

$$
\frac{1}{2}\langle\mu, \varphi\rangle=\lim _{r \downarrow 0} \frac{1}{r^{2}} \int_{\Xi_{r}} u \varphi, \quad \forall \varphi \in C_{0}^{\infty}(\Omega) .
$$

Now (8) follows since, by definition, $\mu=\Delta u-g$. This completes the proof of the theorem.

\section{$5 \quad$ Proof of Theorem 4}

We shall give a proof of Theorem 4 only for the case of codimension $k \geq 3$, the case $k=2$ being entirely analogous.

Using the fact that $u \geq 0$ a.e. in $\Omega$, if $G_{k}$ is the function defined by (36), then we have

$$
\begin{aligned}
\mid \int_{0}^{R} G_{k}(r, t)\left(\int_{\Xi_{t}}\right. & \left.2 u \nabla \varphi \cdot \nabla d+u \varphi a_{0}\right) d t \mid \leq \\
\leq & C \int_{0}^{r}\left(\int_{\Xi_{t}} u\right) d t+C \int_{r}^{R} \frac{r^{k}}{t^{k}}\left(\int_{\Xi_{t}} u\right) d t \\
\leq & C r \int_{\Xi_{r}} u+C \int_{r}^{R} \frac{r^{k}}{t^{k}}\left(\int_{\Xi_{t}} u\right) d t, \quad \forall r \in(0, R) .
\end{aligned}
$$

Choose $R_{1} \in(0, R)$ small so that $C R_{1}<\frac{1}{2}$.

Applying (37) with $R:=R_{1}$ and $\varphi \in C_{0}^{\infty}(\Omega), \varphi \equiv 1$ on $\Xi_{R_{1}}$, then by (61) and our choice of $R_{1}$ we get

$$
\frac{1}{2} \int_{\Xi_{r}} u-C \int_{r}^{R} \frac{r^{k}}{t^{k}}\left(\int_{\Xi_{t}} u\right) d t \leq C r^{2}, \quad \forall r \in\left(0, R_{1}\right) .
$$


We shall use (62) and a bootstrap argument to conclude that

$$
\int_{\Xi_{r}} u \leq C r^{2}, \quad \forall r \in\left(0, R_{1}\right)
$$

In fact, since $\int_{\Xi_{t}} u$ is uniformly bounded for $t \in\left(0, R_{1}\right)$, we have

$$
\int_{r}^{R} \frac{r^{k}}{t^{k}}\left(\int_{\Xi_{t}} u\right) d t \leq C r, \quad \forall r \in\left(0, R_{1}\right) .
$$

In particular, (62) and (64) imply that

$$
\frac{1}{2} \int_{\Xi_{r}} u \leq C r, \quad \forall r \in\left(0, R_{1}\right),
$$

so that

$$
\int_{r}^{R} \frac{r^{k}}{t^{k}}\left(\int_{\Xi_{t}} u\right) d t \leq C r^{2}, \quad \forall r \in\left(0, R_{1}\right) .
$$

Therefore, by (62) and (65), we conclude that estimate (63) holds.

It then follows from (63) and (61), with $R$ replaced by $R_{1}$, that the righthand side in (61) is bounded by $C r^{3}, \forall r \in\left(0, R_{1}\right)$. In particular,

$$
\lim _{r \downarrow 0} \frac{1}{r^{2}}\left\{\int_{0}^{R_{1}} G_{k}(r, t)\left(\int_{\Xi_{t}} 2 u \nabla \varphi \cdot \nabla d+u \varphi a_{0}\right) d t\right\}=0 .
$$

By (37) and (66), we have

$$
-\frac{1}{2(k-2)}\langle\mu, \varphi\rangle=\lim _{r \downarrow 0} \frac{1}{r^{2}} \int_{\Xi_{r}} u \varphi, \quad \forall \varphi \in C_{0}^{\infty}(\Omega) .
$$

If we now apply (67) with estimate (63), we conclude that $\mu$ is a measure. Since $u \geq 0$ a.e. in $\Omega$, then (67) implies that $\mu$ is nonpositive.

\section{Proof of Theorems 5 and 6}

Proof of Theorem 5. We shall split the proof of the theorem into 3 steps:

Step 1. If

$$
\frac{1}{r^{2}} \int_{\Xi_{r}}|u| \quad \text { remains bounded as } \quad r \downarrow 0,
$$


then $\mu$ is a measure and

$$
\langle\mu, \varphi\rangle=-2(k-2) \lim _{r \downarrow 0} \frac{1}{r^{2}} \int_{\Xi_{r}} u \varphi, \quad \forall \varphi \in C_{0}^{\infty}(\Omega) .
$$

Proof. It is easy to see that condition (68) implies that

$$
\lim _{r \downarrow 0} \frac{1}{r^{2}}\left\{\int_{0}^{R_{1}} G_{k}(r, t)\left(\int_{\Xi_{t}} 2 u \nabla \varphi \cdot \nabla d+u \varphi a_{0}\right) d t\right\}=0,
$$

where $G_{k}$ is the function defined by (36). From the limit above and (37), we deduce that (69) holds. In particular, it follows from (68) and (69) that $\mu$ is a measure and

$$
\|\mu\| \leq 2(k-2) \liminf _{r \downarrow 0} \frac{1}{r^{2}} \int_{\Xi_{r}}|u| .
$$

Step 2. If $\mu$ is a measure, then

$$
\frac{1}{r^{2}} \int_{\Xi_{r}}|u| \quad \text { remains bounded as } \quad r \downarrow 0,
$$

and

$$
\|\mu\| \geq 2(k-2) \limsup _{r \downarrow 0} \frac{1}{r^{2}} \int_{\Xi_{r}}|u| .
$$

Proof. In this step we shall use an estimate given in the proof of Theorem 4 and the representation of the solutions of $\Delta v=\nu$ in $\mathbb{R}^{N}$ when $\nu$ is a measure in terms of the fundamental solution. More precisely, let $E(x)=\frac{c_{N}}{|x|^{N-2}}$ be the fundamental solution of $-\Delta$ in $\mathbb{R}^{N}, N \geq 3$, where the constant $c_{N}$ is chosen so that $-\Delta E=\delta_{0}$. If $\nu$ is a Radon measure, then $v:=E * \nu$ satisfies $-\Delta v=\nu$ in $\mathcal{D}^{\prime}\left(\mathbb{R}^{N}\right)$.

Now let $\nu:=g+\mu$ in $\Omega$. Next, we decompose $\nu=\nu^{+}-\nu^{-}$in its positive and negative parts, where $\nu^{ \pm}=g^{ \pm}+\mu^{ \pm}$. Let $v^{ \pm}:=E * \nu^{ \pm}$. As we observed above, we have

$$
-\Delta v^{ \pm}=\nu^{ \pm}=g^{ \pm}+\mu^{ \pm} \quad \text { in } \mathcal{D}^{\prime}\left(\mathbb{R}^{N}\right) .
$$

Moreover, note that $v^{ \pm} \geq 0$ a.e. in $\mathbb{R}^{N}$. In particular, the functions $v^{ \pm}$ satisfy the assumptions of Theorem 4, so that (11) holds with $u$ and $\mu$ replaced by $v^{ \pm}$and $-\mu^{ \pm}$, respectively. In other words, we have

$$
\int_{\Xi_{r}} v^{ \pm} \leq C r^{2}, \quad \forall r \in(0,1),
$$


and

$$
\frac{1}{2(k-2)}\left\langle\mu^{ \pm}, \varphi\right\rangle=\lim _{r \downarrow 0} \frac{1}{r^{2}} \int_{\Xi_{r}} v^{ \pm} \varphi, \quad \forall \varphi \in C_{0}^{\infty}(\Omega) .
$$

On the other hand, it is easy to see that $u=v^{-}-v^{+}+w$ a.e. in $\Omega$ for some harmonic function $w$. Since $w$ is bounded in some neighborhood of $M$, we have

$$
\lim _{r \downarrow 0} \frac{1}{r^{2}} \int_{\Xi_{r}}|w|=0 .
$$

In particular, (71) follows from (73) and (75). Moreover, if we apply (74) with a test function $\varphi$ such that $\varphi \equiv 1$ in some neighborhood of $M$ then we have:

$$
\begin{aligned}
\frac{1}{2(k-2)}\|\mu\| & =\frac{1}{2(k-2)}\left(\left\langle\mu^{+}, 1\right\rangle+\left\langle\mu^{-}, 1\right\rangle+0\right) \\
& =\lim _{r \downarrow 0} \frac{1}{r^{2}} \int_{\Xi_{r}}\left(v^{+}+v^{-}+|w|\right) \geq \limsup _{r \downarrow 0} \frac{1}{r^{2}} \int_{\Xi_{r}}|u| .
\end{aligned}
$$

This concludes the proof of Step 2.

Step 3. Proof of Theorem 5 completed.

By Steps 1 and 2 we know that $\mu$ is a measure if and only if

$$
\frac{1}{r^{2}} \int_{\Xi_{r}}|u| \text { remains bounded as } r \downarrow 0,
$$

in which case formula (14) holds. Moreover, applying (70) and (72) we get

$$
\|\mu\| \leq 2(k-2) \liminf _{r \downarrow 0} \frac{1}{r^{2}} \int_{\Xi_{r}}|u| \leq 2(k-2) \limsup _{r \downarrow 0} \frac{1}{r^{2}} \int_{\Xi_{r}}|u| \leq\|\mu\|,
$$

so that all the inequalities are reduced to equalities in the estimate above and (15) holds.

Proof of Theorem 6. The proof of Theorem 6 follows along the same lines as those in the previous one and shall be omitted.

Remark 8 Although we derived (14) in Theorem 5 through a somewhat lengthy computation, there is a more natural approach if one assumes that the limits involved exist. Indeed, take $\varphi \in C_{0}^{\infty}(\Omega)$. Then, using l'Hôpital's rule

$$
\lim _{r \downarrow 0} \frac{1}{r^{2}} \int_{\Xi_{r}} u \varphi=\lim _{r \downarrow 0} \frac{1}{2 r} \int_{\partial \Xi_{r}} u \varphi .
$$


But, using formula (106) of the Appendix (with $\lambda=1$ )

$$
\begin{aligned}
& \lim _{r \downarrow 0} \frac{1}{r} \int_{\partial \Xi_{r}} u \varphi= \lim _{r \downarrow 0} \frac{1}{r}\left\{r^{k-1} \int_{\partial \Xi_{1}}(u \varphi) \circ \pi_{r} \Theta(\xi, r) d \sigma(\xi)\right\} \\
&=\lim _{r \downarrow 0}\left\{r^{k-1} \int_{\partial \Xi_{1}} \frac{\partial(u \varphi)}{\partial \nu} \circ \pi_{r} \Theta+r^{k-1} \int_{\partial \Xi_{1}}(u \varphi) \circ \pi_{r} \frac{\partial \Theta}{\partial r}\right. \\
&\left.+(k-1) r^{k-2} \int_{\partial \Xi_{1}}(u \varphi) \circ \pi_{r} \Theta\right\} \\
&=\lim _{r \downarrow 0}\left\{\int_{\partial \Xi_{r}} \frac{\partial(u \varphi)}{\partial \nu}+\int_{\partial \Xi_{r}} u \varphi\left(\frac{\partial \Theta}{\partial r} \frac{1}{\Theta}\right) \circ \pi_{1}\right. \\
&\left.+\frac{k-1}{r} \int_{\partial \Xi_{r}} u \varphi\right\} .
\end{aligned}
$$

We can solve from the previous equations for $\lim _{r \downarrow 0} \frac{1}{r} \int_{\partial \Xi_{r}} u \varphi$ :

$$
\lim _{r \downarrow 0} \frac{1}{r} \int_{\partial \Xi_{r}} u \varphi=-\frac{1}{(k-2)} \lim _{r \downarrow 0}\left\{\int_{\partial \Xi_{r}} \frac{\partial(u \varphi)}{\partial \nu}+\int_{\partial \Xi_{r}} u \varphi\left(\frac{\partial \Theta}{\partial r} \frac{1}{\Theta}\right) \circ \pi_{1}\right\}
$$

and, integrating by parts and using estimates in the Appendix, we find

$$
\lim _{r \downarrow 0} \frac{1}{r} \int_{\partial \Xi_{r}} u \varphi=-\frac{1}{(k-2)}\langle\mu, \varphi\rangle .
$$

Thus, (76) and (77) combined yield

$$
\lim _{r \downarrow 0} \frac{1}{r^{2}} \int_{\Xi_{r}} u \varphi=-\frac{1}{2(k-2)}\langle\mu, \varphi\rangle .
$$

Remark 9 Let us mention that formula (14) in Theorem 5 holds under weaker conditions than the one mentioned in that theorem, namely that $\frac{1}{r^{2}} \int_{\Xi_{r}}|u|$ remains bounded as $r \downarrow 0$ or equivalently, that $\Delta u=\mu+g$ with $g \in L_{\mathrm{loc}}^{1}(\Omega)$ and $\mu$ a Radon measure supported in $M$. For example, it is easy to check that if

$$
\frac{1}{r} \int_{\Xi_{r}}|u| \rightarrow 0 \quad \text { as } r \downarrow 0
$$

then (14) holds, i.e. (in codimension $k \geq 3$ ),

$$
\lim _{r \downarrow 0} \frac{1}{r^{2}} \int_{\Xi_{r}} u \varphi=-\frac{1}{2(k-2)}\langle\mu, \varphi\rangle \quad \forall \varphi \in C_{0}^{\infty}(\Omega) .
$$


This suggests the following

Open problem. Let $\Omega \subset \mathbb{R}^{N}$ be a bounded open set and let $M \subset \Omega$ be a compact, smooth manifold without boundary of codimension $k \geq 3$. Let $u \in$ $L_{\text {loc }}^{1}(\Omega)$ and assume there exists $g \in L_{\text {loc }}^{1}(\Omega)$ such that $\Delta u=g$ in $\mathcal{D}^{\prime}(\Omega \backslash M)$. Set $\mu:=\Delta u-g$ in $\mathcal{D}^{\prime}(\Omega)$. Assume that

$$
\lim _{r \downarrow 0} \frac{1}{r} \int_{\Xi_{r}}|u|=0 .
$$

Is $\mu$ a measure?

The requirement (78) cannot be further relaxed, for instance, by asking instead that

$$
\frac{1}{r} \int_{\Xi_{r}}|u| \text { remains bounded as } r \downarrow 0 \text {. }
$$

For example, if $u(x)=\frac{x_{1}}{|x|^{3}}$ in $\mathbb{R}^{3}$, then $\Delta u=c D_{x_{1}} \delta_{0}$ for some constant $c \neq 0$, and $\frac{1}{r} \int_{\Xi_{r}}|u|$ remains bounded away from 0 as $r \downarrow 0$. In any case, if (79) holds, then from the formulas in Lemma 11 we see that $\mu$ has to be a distribution of order 1 .

\section{$7 \quad$ Proof of Theorem 8}

As in $[\mathrm{BrL}]$, we shall prove the following 2 steps:

Step 1. $u \in L_{\text {loc }}^{1}(\Omega)$ and there exists a constant $C>0$ such that

$$
\int_{\Xi_{r}} u \leq C \begin{cases}r^{2} & \text { if } k \geq 3, \\ r^{2} \log \frac{1}{r} & \text { if } k=2,\end{cases}
$$

for all $r>0$ sufficiently small.

Step 2. Set $h:=-\Delta u$ a.e. in $\Omega \backslash M$. Then $h \in L_{\mathrm{loc}}^{1}(\Omega)$ and

$$
\int_{\Omega} h \varphi \leq-\int_{\Omega} u \Delta \varphi, \quad \forall \varphi \in \mathcal{F}
$$

where the class $\mathcal{F}$ of admissible test functions is given as follows:

$$
\mathcal{F}:=\left\{\begin{array}{l|l}
\varphi \in C_{0}^{\infty}(\Omega) & \begin{array}{l}
\varphi \geq 0 \text { in } \Omega, \\
\exists \lambda>0 \text { such that } \nabla \varphi \cdot \nabla d=0 \text { on } \Xi_{\lambda}
\end{array}
\end{array}\right\} .
$$


By Steps 1 and 2, we conclude that $u \in L_{\text {loc }}^{1}(\Omega)$, and we can write

$$
-\Delta u=h+\mu \quad \text { in } \mathcal{D}^{\prime}(\Omega)
$$

for some function $h \in L_{\mathrm{loc}}^{1}(\Omega)$ and some distribution $\mu$ supported on $M$. Since $u \geq 0$ a.e. in $\Omega$, we deduce from Theorem 4 that $\mu$ is a positive measure (note the change of sign in the definition of $\mu$ ). In other words, in order to show that Theorem 8 holds, it suffices to prove Steps 1 and 2.

The details go as follows:

Proof of Step 1. Consider the function

$$
\bar{u}(r)=\frac{1}{r^{k-1}} \int_{\partial \Xi_{r}} u d \sigma=\int_{\partial \Xi_{1}} u \circ \pi_{r} \Theta(\sigma, r) d \sigma, \quad 0<r<1,
$$

where $\Theta$ is a smooth function defined on $\partial \Xi_{1} \times[0,1]$ which arises from the change of variables (see (107) and Lemma 12 in the Appendix), and $\pi_{r}$ is defined by

$$
\pi_{r}(x)=\pi(x)+r \frac{x-\pi(x)}{d(x)}, \quad x \in \Xi_{2} \backslash M .
$$

We will use the function $\bar{u}$ to prove (80) in a similar way as in BrezisLions $[\mathrm{BrL}]$. In order to get some of its properties, suppose for a moment that $u \in C^{\infty}(\Omega \backslash M)$; then

$$
\frac{d \bar{u}}{d r}(r)=\int_{\partial \Xi_{1}} \frac{\partial u}{\partial \nu} \circ \pi_{r} \Theta(\xi, r) d \sigma(\xi)+\int_{\partial \Xi_{1}} u \circ \pi_{r} \frac{\partial \Theta}{\partial r}(\xi, r) d \sigma(\xi) .
$$

Hence, by Corollary 13,

$$
r^{k-1} \frac{d \bar{u}}{d r}(r)=\int_{\partial \Xi_{r}} \frac{\partial u}{\partial \nu} d \sigma+r^{k-1} \int_{\partial \Xi_{1}} u \circ \pi_{r} \frac{\partial \Theta}{\partial r}(\xi, r) d \sigma(\xi),
$$

and integrating by parts

$$
\begin{aligned}
=-\int_{\Xi_{r_{0}} \backslash \Xi_{r}} \Delta u & +\int_{\partial \Xi_{r_{0}}} \frac{\partial u}{\partial \nu} d \sigma+ \\
& +r^{k-1} \int_{\partial \Xi_{1}} u \circ \pi_{r} \frac{\partial \Theta}{\partial r}(\xi, r) d \sigma(\xi),
\end{aligned}
$$

for any $r_{0}>0$ small enough. Throughout the step, we will always denote by $\nu$ the unit normal vector to $\Xi_{r}$, pointing out of $\Xi_{r}$ (which explains the minus sign in front of $\int_{\Xi_{r_{0}} \backslash \Xi_{r}} \Delta u$ in the expression above). 
For a general $u \in L_{\text {loc }}^{1}(\Omega \backslash M)$ with $\Delta u \in L_{\text {loc }}^{1}(\Omega \backslash M)$, by using Fubini's Theorem and the fact that $u \in W_{\text {loc }}^{1,1}(\Omega \backslash M)$, it follows by density that $\bar{u} \in C^{1}(0,1), d \bar{u} / d r$ is absolutely continuous on $(0,1)$, and

$$
r^{k-1} \frac{d \bar{u}}{d r}(r)=-\int_{\Xi_{r_{0}} \backslash \Xi_{r}} \Delta u+\int_{\partial \Xi_{r_{0}}} \frac{\partial u}{\partial \nu} d \sigma+r^{k-1} \int_{\partial \Xi_{1}} u \circ \pi_{r} \frac{\partial \Theta}{\partial r}(\xi, r) d \sigma(\xi),
$$

is still true for a.e. $r_{0}>0$ small (which will be fixed later).

We now proceed with the main computation. The next formulas hold for a.e. $r \in(0,1)$.

$$
\begin{aligned}
\frac{1}{r^{k-1}} \frac{d}{d r}\left(r^{k-1} \frac{d \bar{u}}{d r}\right)= & \frac{1}{r^{k-1}} \int_{\partial \Xi_{r}} \Delta u \\
& \quad+\frac{1}{r^{k-1}} \frac{d}{d r}\left\{r^{k-1} \int_{\partial \Xi_{1}} u \circ \pi_{r} \frac{\partial \Theta}{\partial r}(\xi, r) d \sigma(\xi)\right\} \\
= & \frac{1}{r^{k-1}} \int_{\partial \Xi_{r}} \Delta u+\frac{k-1}{r} I_{1}+I_{2},
\end{aligned}
$$

where

$$
I_{1}=\int_{\partial \Xi_{1}} u \circ \pi_{r} \frac{\partial \Theta}{\partial r}(\xi, r) d \sigma(\xi)=\frac{1}{r^{k-1}} \int_{\partial \Xi_{r}} u\left(\frac{1}{\Theta} \frac{\partial \Theta}{\partial r}\right) \circ \pi_{1}
$$

and

$$
\begin{aligned}
I_{2} & =r^{k-1} \frac{d}{d r} \int_{\partial \Xi_{1}} u \circ \pi_{r} \frac{\partial \Theta}{\partial r}(\xi, r) d \sigma(\xi) \\
& =r^{k-1} \int_{\partial \Xi_{1}} \frac{\partial u}{\partial \nu} \circ \pi_{r} \frac{\partial \Theta}{\partial r}+r^{k-1} \int_{\partial \Xi_{1}} u \circ \pi_{r} \frac{\partial^{2} \Theta}{\partial r^{2}} \\
& =\int_{\partial \Xi_{r}} \frac{\partial u}{\partial \nu}\left(\frac{1}{\Theta} \frac{\partial \Theta}{\partial r}\right) \circ \pi_{1}+\int_{\partial \Xi_{r}} u\left(\frac{1}{\Theta} \frac{\partial^{2} \Theta}{\partial r^{2}}\right) \circ \pi_{1} .
\end{aligned}
$$

At this point it is convenient to introduce some notation:

$$
\vartheta^{(i)}(x)=\frac{1}{\Theta\left(\pi_{1}(x), d(x)\right)} \frac{\partial^{i} \Theta}{\partial r^{i}}\left(\pi_{1}(x), d(x)\right), \quad i=1,2 .
$$

Then we can rewrite $I_{1}$ and $I_{2}$ as

$$
\begin{aligned}
I_{1} & =\frac{1}{r^{k-1}} \int_{\partial \Xi_{r}} u \vartheta^{(1)}, \\
I_{2} & =\int_{\partial \Xi_{r}} \frac{\partial u}{\partial \nu} \vartheta^{(1)}+\int_{\partial \Xi_{r}} u \vartheta^{(2)} .
\end{aligned}
$$


Integrating by parts the expression for $I_{2}$, we get

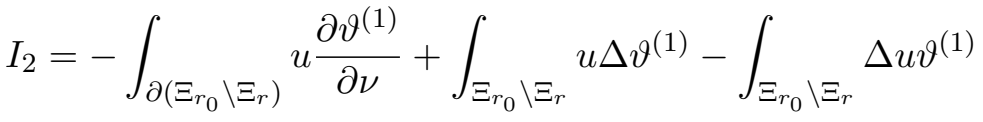

$$
\begin{aligned}
& +\int_{\partial \Xi_{r_{0}}} \frac{\partial u}{\partial \nu} \vartheta^{(1)}+\int_{\partial \Xi_{r}} u \vartheta^{(2)} .
\end{aligned}
$$

From Corollary 13 in the Appendix (in combination with the lower bound for $\Theta$ of Lemma 9) we obtain the following estimates for $\vartheta^{(i)}$

$$
\begin{aligned}
\left|D^{j} \vartheta^{(1)}\right| & \leq C d^{k-j-1}, \quad j=0,1,2 ; \\
\left|\vartheta^{(2)}\right| & \leq C d^{k-2} .
\end{aligned}
$$

Therefore

$$
I_{1}=\frac{1}{r^{k-1}} \int_{\partial \Xi_{r}} u \vartheta^{(1)} \leq C \int_{\partial \Xi_{r}} u
$$

and

$$
I_{2} \leq C \int_{\partial \Xi_{r}} u+C \int_{\Xi_{r_{0}} \backslash \Xi_{r}} u d^{k-3}-\int_{\Xi_{r_{0}} \backslash \Xi_{r}} \Delta u \vartheta^{(1)}+C .
$$

Combining (83), (88) and (89) we find

$$
\begin{aligned}
\frac{1}{r^{k-1}} \frac{d}{d r}\left(r^{k-1} \frac{d \bar{u}}{d r}\right) \leq & \frac{1}{r^{k-1}} \int_{\partial \Xi_{r}} \Delta u+\frac{C}{r} \int_{\partial \Xi_{r}} u+C \int_{\partial \Xi_{r}} u+ \\
& +C \int_{\Xi_{r_{0}} \backslash \Xi_{r}} u d^{k-3}-\int_{\Xi_{r_{0}} \backslash \Xi_{r}} \Delta u \vartheta^{(1)}+C .
\end{aligned}
$$

Then, multiplying the last inequality by $r^{k-1}$ and integrating with respect to $r$ yields

$$
\begin{aligned}
r_{0}^{k-1} \frac{d \bar{u}}{d r}\left(r_{0}\right)- & r^{k-1} \frac{d \bar{u}}{d r}(r) \leq \\
\leq & C \int_{r}^{r_{0}} s^{k-2} \int_{\partial \Xi_{s}} u d \sigma d s+C \int_{r}^{r_{0}} s^{k-1} \int_{\partial \Xi_{s}} u d \sigma d s \\
& +C \int_{r}^{r_{0}} s^{k-1} \int_{\Xi_{r_{0}} \backslash \Xi_{s}} u d^{k-3} d \sigma d s \\
& +\int_{r}^{r_{0}} \int_{\partial \Xi_{s}} \Delta u d \sigma d s-\int_{r}^{r_{0}} s^{k-1} \int_{\Xi_{r_{0}} \backslash \Xi_{s}} \Delta u \vartheta^{(1)} d x d s \\
& +C r_{0}^{k} .
\end{aligned}
$$


We now estimate each term on the right-hand side of (90). We start with

$$
\int_{r}^{r_{0}} s^{k-2} \int_{\partial \Xi_{s}} u d \sigma d s \leq r_{0}^{k-2} \int_{r}^{r_{0}} \int_{\partial \Xi_{s}} u d \sigma d s \leq \int_{r}^{r_{0}} s^{k-1} \bar{u}(s) d s .
$$

Similarly,

$$
\int_{r}^{r_{0}} s^{k-1} \int_{\partial \Xi_{s}} u d \sigma d s \leq \int_{r}^{r_{0}} s^{k-1} \bar{u}(s) d s .
$$

The third term on the right-hand side of (90) is, using Fubini,

$$
\begin{aligned}
\int_{r}^{r_{0}} s^{k-1} \int_{\Xi_{r_{0}} \backslash \Xi_{s}} u d^{k-3} d \sigma d s & =\int_{r}^{r_{0}} \int_{s}^{r_{0}} \int_{\partial \Xi_{\lambda}} s^{k-1} u \lambda^{k-3} d \sigma d \lambda d s \\
& =\int_{r}^{r_{0}} \int_{\partial \Xi_{\lambda}} u \lambda^{k-3} \int_{r}^{\lambda} s^{k-1} d s d \sigma d \lambda \\
& \leq C \int_{r}^{r_{0}} \int_{\partial \Xi_{\lambda}} u \lambda^{2 k-3} d \sigma d \lambda \\
& \leq C \int_{r}^{r_{0}} s^{k-1} \bar{u}(s) d s,
\end{aligned}
$$

since $\lambda^{2 k-3} \leq 1$, for $0<\lambda \leq r_{0} \leq 1$. We now estimate the fifth term in (90) using Fubini again:

$$
\begin{aligned}
\int_{r}^{r_{0}} s^{k-1} \int_{\Xi_{r_{0}} \backslash \Xi_{s}} \Delta u \vartheta^{(1)} d \sigma d s & =\int_{r}^{r_{0}} \int_{s}^{r_{0}} \int_{\partial \Xi_{\lambda}} s^{k-1} \Delta u \vartheta^{(1)} \lambda^{k-1} d \sigma d \lambda d s \\
& =\int_{r}^{r_{0}} \int_{\partial \Xi_{\lambda}} \Delta u \vartheta^{(1)} \lambda^{k-1} \int_{r}^{\lambda} s^{k-1} d s d \sigma d \lambda \\
& =\int_{r}^{r_{0}} \int_{\partial \Xi_{\lambda}} \Delta u O\left(r_{0}^{2 k-1}\right) d \sigma d \lambda,
\end{aligned}
$$

where $O\left(r_{0}^{2 k-1}\right)$ denotes a function bounded by $C r_{0}^{2 k-1}$. Hence the fourth and fifth terms of (90) combined yield

$$
\begin{aligned}
\int_{r}^{r_{0}} \int_{\partial \Xi_{s}} \Delta u d \sigma d s-\int_{r}^{r_{0}} s^{k-1} \int_{\Xi_{r_{0}} \backslash \Xi_{s}} \Delta u \vartheta^{(1)} d \sigma d s= \\
=\int_{r}^{r_{0}} \int_{\partial \Xi_{\lambda}} \Delta u\left(1+O\left(r_{0}^{2 k-1}\right)\right) d \sigma d \lambda .
\end{aligned}
$$


We now fix $r_{0}>0$ small enough so that $1 / 2 \leq 1+O\left(r_{0}^{2 k-1}\right) \leq 3 / 2$. Since $\Delta u \leq a u+f$ a.e. in $\Omega \backslash M$, we conclude that

$$
\begin{aligned}
& \int_{r}^{r_{0}} \int_{\partial \Xi_{s}} \Delta u d \sigma d s-\int_{r}^{r_{0}} s^{k-1} \int_{\Xi_{\Xi_{0}} \backslash \Xi_{s}} \Delta u \vartheta^{(1)} d \sigma d s \leq \\
& \leq C \int_{r}^{r_{0}} \int_{\partial \Xi_{\lambda}} a u+f d \sigma d \lambda \\
& \leq C \int_{r}^{r_{0}} s^{k-1} \bar{u}(s) d s+\int_{\Xi_{\lambda}} f
\end{aligned}
$$

(in the hypotheses of the theorem, after replacing $f$ with $f^{+}$we may assume that $f \geq 0$ a.e. in $\Omega$ ). Hence, from (90)-(94), we get the estimate

$$
-r^{k-1} \frac{d \bar{u}}{d r}(r) \leq C \int_{r}^{r_{0}} s^{k-1} \bar{u}(s) d s+C .
$$

We now proceed exactly as in $[\mathrm{BrL}]$. Let $0<R<r_{0}$ to be chosen later and define

$$
\psi_{R}(r):=\int_{r}^{R} s^{k-1} \bar{u}(s) d s, \quad 0<r<R .
$$

With this notation we have

$$
-r^{k-1} \frac{d \bar{u}}{d r}(r) \leq C \psi_{R}(r)+C_{R}
$$

where $C_{R}$ is a constant that depends on $R$, but $C$ is independent of $R$. After integration we find

$$
\bar{u}(r)-\bar{u}(R) \leq C \int_{r}^{R} \frac{\psi(s)}{s^{k-1}} d s+C_{R} \int_{r}^{R} \frac{d s}{s^{k-1}},
$$

and therefore

$$
\bar{u}(r) \leq C \int_{r}^{R} \frac{1}{s^{k-1}} \psi_{R}(s) d s+C_{R}\left(1+\frac{1}{r^{k-2}}\right)
$$

if $k \geq 3$ and, in the case $k=2$, we have to replace $1 / r^{k-2}$ by $|\log r|$ in the second term on the right-hand side. Since $\psi_{R}$ is nonincreasing we obtain thus

$$
r^{k-1} \bar{u}(r) \leq C R \psi_{R}(r)+C_{R}
$$

Integrating once more we get

$$
\begin{aligned}
\psi_{R}(r)=\int_{r}^{R} s^{k-1} \bar{u}(s) d s & \leq C R \int_{r}^{R} \psi_{R}(s) d s+C_{R} \\
& \leq C R^{2} \psi_{R}(r)+C_{R} .
\end{aligned}
$$


We now choose $0<R<r_{0}$ such that $\left(1-C R^{2}\right) \geq 1 / 2$, so that from (96) we see that

$$
\psi_{R}(r) \leq C,
$$

with $C$ independent of $r \in(0, R)$. By letting $r \rightarrow 0$ we conclude that $u \in L_{\text {loc }}^{1}(\Omega)$. Moreover, from (95) we see that

$$
\bar{u}(r) \leq C \begin{cases}\frac{1}{r^{k-2}} & \text { if } k \geq 3 \\ |\log r| & \text { if } k=2\end{cases}
$$

which implies the estimate

$$
\int_{\Xi_{r}} u=\int_{0}^{r} s^{k-1} \bar{u}(s) d s \leq C \begin{cases}r^{2} & \text { if } k \geq 3, \\ r^{2}|\log r| & \text { if } k=2 .\end{cases}
$$

This concludes the proof of the first step.

Proof of Step 2. First, note that to prove the whole statement of Step 2, it is enough to show that (81) holds. In fact, suppose that (81) has already been established. By the assumptions of the theorem, we know that $h \geq-a u-f$ a.e. in $\Omega$, and $a u+f \in L_{\text {loc }}^{1}(\Omega)$ by Step 1. If we take an admissible test function $\varphi \in \mathcal{F}$ such that $\varphi \equiv 1$ in some small neighborhood of $M$, then we have

$$
0 \leq \int_{\Omega}(h+a u+f) \varphi \leq-\int_{\Omega} u \Delta \varphi+\int_{\Omega}(a u+f) \varphi<\infty,
$$

which implies that $h \in L_{\text {loc }}^{1}(\Omega)$.

We now proceed with the proof of (81).

Let $\varphi \in \mathcal{F}$. Since (81) is trivially satisfied if $\varphi \equiv 0$ near $M$ (in fact, we have equality in (81) in this case), there is no loss of generality if we assume that $\operatorname{supp} \varphi \subset \Xi_{1}$ and $\varphi \not \equiv 0$ near $M$. Next, fix a $\lambda>0$ such that $\nabla \varphi \cdot \nabla d=0$ in $\Xi_{\lambda}$.

Let $\Phi \in C^{3}(\mathbb{R})$ be a convex function such that $\Phi(t)=0$ for $t \geq 1$, and $\Phi(0)=1$, to be given explicitly below.

For $0<\varepsilon<1$, and if $k \geq 3$, define

$$
\varphi_{\varepsilon}(x):= \begin{cases}\varphi(x) \Phi\left(\frac{\varepsilon^{k-2}}{d(x)^{k-2}}\right) & \text { if } x \in \Xi_{1} \backslash \Xi_{\varepsilon} \\ 0 & \text { otherwise }\end{cases}
$$


if $k=2$, we let

$$
\varphi_{\varepsilon}(x):=\left\{\begin{array}{ll}
\varphi(x) \Phi\left(\frac{\log \frac{1}{d(x)}}{\log \frac{1}{\varepsilon}}\right) & \text { if } x \in \Xi_{1} \backslash \Xi_{\varepsilon} . \\
0 & \text { otherwise }
\end{array} .\right.
$$

By construction, we have $\varphi_{\varepsilon} \in C_{0}^{3}(\Omega)$ and $\varphi_{\varepsilon} \equiv 0$ on $\Xi_{\varepsilon}$. In particular,

$$
\int_{\Omega} h \varphi_{\varepsilon}=-\int_{\Omega} \Delta u \varphi_{\varepsilon}=-\int_{\Omega} u \Delta \varphi_{\varepsilon}
$$

In the argument that follows, we assume $k \geq 3$, the proof of (81) when $k=2$ being entirely analogous.

If we compute $\Delta \varphi_{\varepsilon}$ explicitly on $\Xi_{1} \backslash \Xi_{\varepsilon}$, and using (34) and (35), we get (recall that $\varphi_{\varepsilon} \equiv 0$ outside this set)

$$
\begin{aligned}
\Delta \varphi_{\varepsilon}=\Delta & \varphi \Phi \\
& \left(\frac{\varepsilon^{k-2}}{d^{k-2}}\right)-2(k-2) \nabla \varphi \cdot \nabla d \Phi^{\prime}\left(\frac{\varepsilon^{k-2}}{d^{k-2}}\right) \frac{\varepsilon^{k-2}}{d^{k-1}}+ \\
& +\varphi \frac{\varepsilon^{k-2}}{d^{k-1}}\left\{(k-2)^{2} \Phi^{\prime \prime}\left(\frac{\varepsilon^{k-2}}{d^{k-2}}\right) \frac{\varepsilon^{k-2}}{d^{k-1}}+\Phi^{\prime}\left(\frac{\varepsilon^{k-2}}{d^{k-2}}\right) O(1)\right\},
\end{aligned}
$$

where $O(1)$ is a quantity which remains bounded as $\varepsilon \downarrow 0$.

Note that

$$
\Phi\left(\frac{\varepsilon^{k-2}}{d^{k-2}}\right) \rightarrow \Phi(0)=1
$$

and

$$
\Phi^{\prime}\left(\frac{\varepsilon^{k-2}}{d^{k-2}}\right) \frac{\varepsilon^{k-2}}{d^{k-1}} \rightarrow 0
$$

both limits being uniform in any compact subset of $\Omega \backslash M$ as $\varepsilon \downarrow 0$.

Since $\Delta \varphi \Phi\left(\frac{\varepsilon^{k-2}}{d^{k-2}}\right)$ is uniformly bounded and $\nabla \varphi \cdot \nabla d=0$ on $\Xi_{\lambda}$, we conclude that

$$
\begin{aligned}
\int_{\Omega}\left[\Delta \varphi \Phi\left(\frac{\varepsilon^{k-2}}{d^{k-2}}\right)-2(k-2) \nabla \varphi \cdot \nabla d \Phi^{\prime}\left(\frac{\varepsilon^{k-2}}{d^{k-2}}\right)\right. & \left.\frac{\varepsilon^{k-2}}{d^{k-1}}\right] u \\
& \longrightarrow \int_{\Omega} u \Delta \varphi \quad \text { as } \varepsilon \downarrow 0 .
\end{aligned}
$$


Next, we shall analyze the behavior of the term between brackets in (98).

Before that, let us make a special choice of the function $\Phi$.

Let $a \geq 3$ be a number sufficiently large to be chosen below. Take $\Phi: \mathbb{R} \rightarrow \mathbb{R}$ to be defined as

$$
\Phi(t):=\left\{\begin{array}{ll}
(1-t)^{a+1} & \text { if } t \leq 1 \\
0 & \text { otherwise }
\end{array} .\right.
$$

In particular, $\Phi \in C^{3}(\mathbb{R}), \Phi(t)=0$ for $t \geq 1$, and $\Phi(0)=1$.

For $x \in \Xi_{1} \backslash \Xi_{\varepsilon}$, we have

$$
\begin{aligned}
& \frac{\varepsilon^{k-2}}{d^{k-1}}\left\{(k-2)^{2} \Phi^{\prime \prime}\left(\frac{\varepsilon^{k-2}}{d^{k-2}}\right) \frac{\varepsilon^{k-2}}{d^{k-1}}+\Phi^{\prime}\left(\frac{\varepsilon^{k-2}}{d^{k-2}}\right) O(1)\right\}= \\
& =(a+1) \frac{\varepsilon^{k-2}}{d^{k-1}}\left(1-\frac{\varepsilon^{k-2}}{d^{k-2}}\right)^{a-1}\left\{a(k-2)^{2} \frac{\varepsilon^{k-2}}{d^{k-1}}-\left(1-\frac{\varepsilon^{k-2}}{d^{k-2}}\right) O(1)\right\} \\
& =(a+1) \frac{\varepsilon^{k-2}}{d^{k-1}}\left(1-\frac{\varepsilon^{k-2}}{d^{k-2}}\right)^{a-1}\left\{\frac{\varepsilon^{k-2}}{d^{k-1}}\left(a(k-2)^{2}+O(1) d\right)-O(1)\right\} .
\end{aligned}
$$

Now choose $K>0$ and then $a \geq 3$ both large enough so that

$$
\frac{a(k-2)^{2}}{2} \geq K \geq|O(1)|, \quad \text { for } 0<\varepsilon<1 .
$$

Then we get

$$
\begin{aligned}
& \frac{\varepsilon^{k-2}}{d^{k-1}}\left\{(k-2)^{2} \Phi^{\prime \prime}\left(\frac{\varepsilon^{k-2}}{d^{k-2}}\right) \frac{\varepsilon^{k-2}}{d^{k-1}}+\Phi^{\prime}\left(\frac{\varepsilon^{k-2}}{d^{k-2}}\right) O(1)\right\}= \\
& \quad \geq(a+1) \frac{\varepsilon^{k-2}}{d^{k-1}}\left(1-\frac{\varepsilon^{k-2}}{d^{k-2}}\right)^{a-1}\left\{\frac{a(k-2)^{2}}{2} \frac{\varepsilon^{k-2}}{d^{k-1}}-O(1)\right\}=: H
\end{aligned}
$$

Next we split the estimate for a lower bound of $H$ into 2 cases, depending on how near the point $x$ is with respect to the singular set $M$ :

Case 1. $\frac{a(k-2)^{2}}{2} \frac{\varepsilon^{k-2}}{d^{k-1}} \geq K$.

In this case, by our very choice of $K$, the expression defining $H$ must be nonnegative, i.e., $H \geq 0$.

Case 2. $\frac{a(k-2)^{2}}{2} \frac{\varepsilon^{k-2}}{d^{k-1}}<K$. 
If the inequality above holds, we have

$$
\begin{aligned}
H & \geq-(a+1) \frac{\varepsilon^{k-2}}{d^{k-1}}\left(1-\frac{\varepsilon^{k-2}}{d^{k-2}}\right)^{a-1} K \\
& \geq-(a+1) \frac{2 K}{a(k-2)^{2}} K>-\frac{8}{3} K^{2}=:-C .
\end{aligned}
$$

In both cases, we have

$$
H \geq-C,
$$

for some constant $C>0$ independent of $\varepsilon$ and $\varphi$.

It now follows from (98)-(100) and Fatou's Lemma (recall that $h \geq-a u-$ $\left.f \in L_{\mathrm{loc}}^{1}(\Omega)\right)$ that, if we let $\varepsilon \downarrow 0$ in (97), we get

$$
\int_{\Omega} h \varphi \leq-\int_{\Omega} \Delta u \varphi+C \int_{\Omega} u \varphi, \quad \forall \varphi \in \mathcal{F}
$$

which is "almost" the inequality we want to prove. In any case, the same argument we presented in the beginning of this step, applied to (101), already gives that $h \in L_{\text {loc }}^{1}(\Omega)$. Next, we show how the constant $C>0$ above can be removed.

Given any $\delta>0$ small, let $\eta_{\delta} \in C_{0}^{\infty}\left(\Xi_{\delta}\right)$ be such that $0 \leq \eta_{\delta} \leq 1$ and $\eta_{\delta} \equiv 1$ on $\Xi_{\delta / 2}$. Note that $\varphi \eta_{\delta}$ still belongs to $\mathcal{F}$ so that, after replacing $\varphi$ in (101) by $\varphi \eta_{\delta}$, we get

$$
\int_{\Omega} h \varphi \eta_{\delta} \leq-\int_{\Omega} u \Delta\left(\varphi \eta_{\delta}\right)+C \int_{\Omega} u \varphi \eta_{\delta}
$$

On the other hand, since $\varphi\left(1-\eta_{\delta}\right) \in C_{0}^{\infty}(\Omega \backslash M)$,

$$
\int_{\Omega} h \varphi\left(1-\eta_{\delta}\right)=-\int_{\Omega} u \Delta\left(\varphi\left(1-\eta_{\delta}\right)\right) .
$$

Now adding both relations, we obtain

$$
\int_{\Omega} h \varphi \leq-\int_{\Omega} u \Delta \varphi+C \int_{\Omega} u \varphi \eta_{\delta}
$$

If we let $\delta \downarrow 0$ in the inequality above, we get (81), as claimed. This concludes the proof of Step 2. 


\section{Proof of Corollary 10}

Let $u \in L_{\text {loc }}^{1}(\Omega), u \geq 0$ a.e. in $\Omega$, be as in Corollary 9. Since $f(u) \in L_{\text {loc }}^{1}(\Omega)$, (25) implies that

$$
\begin{aligned}
u \in L_{\mathrm{loc}}^{\frac{k}{k-2}}(\Omega) & \text { if } k>2, \\
\mathrm{e}^{a u} \in L_{\text {loc }}^{1}(\Omega) & \text { if } k=2, \text { for all } a>0 .
\end{aligned}
$$

If $k>2$, we apply Hölder's inequality to conclude from (102) (using the fact that $\left|\Xi_{r}\right| \sim r^{k}$ as $r \downarrow 0$ ) that

$$
\lim _{r \downarrow 0} \frac{1}{r^{2}} \int_{\Xi_{r}} u=0 .
$$

By Corollary 7 , we must have $\mu=0$ in (24), which proves the result in the case $k \geq 3$.

Let us now suppose $k=2$. For $a>0$ fixed, we have by Jensen's inequality and (103) that

$$
\mathrm{e}^{\frac{1}{\Xi_{r} \mid} \int_{\Xi_{r}} a u} \leq \frac{1}{\left|\Xi_{r}\right|} \int_{\Xi_{r}} \mathrm{e}^{a u} \leq \frac{C_{a}}{\left|\Xi_{r}\right|}, \quad \forall r>0 \text { small, }
$$

where $C_{a}>0$ is a constant depending on $a$. We conclude that

$$
\frac{1}{\left|\Xi_{r}\right|} \int_{\Xi_{r}} a u \leq \log \frac{C_{a}}{\left|\Xi_{r}\right|}
$$

Let $0<\alpha_{1} \leq \alpha_{2}$ be such that $\alpha_{1} r^{2} \leq\left|\Xi_{r}\right| \leq \alpha_{2} r^{2}$ for all $r>0$ small. From (104) we get

$$
\frac{1}{\alpha_{2} r^{2} \log 1 / r} \int_{\Xi_{r}} a u \leq \frac{\log \left(C_{a} / \alpha_{1} r^{2}\right)}{\log 1 / r}=2+\frac{\log \left(C_{a} / \alpha_{1}\right)}{\log 1 / r} .
$$

By letting $r \downarrow 0$ we deduce that

$$
\limsup _{r \downarrow 0} \frac{1}{r^{2}|\log r|} \int_{\Xi_{r}} u \leq \frac{2 \alpha_{2}}{a}, \quad \forall a>0 .
$$

If we take $a \uparrow \infty$, then we have

$$
\lim _{r \downarrow 0} \frac{1}{r^{2}|\log r|} \int_{\Xi_{r}} u=0 .
$$

We now invoke Corollary 7 to get the result in the case $k=2$. 


\section{Appendix}

In the sequel, we shall assume that $\Xi_{r}$ is a tubular neighborhood of $M^{N-k}$ of radius $r$, where $M^{N-k}$ is a compact manifold without boundary in $\mathbb{R}^{N}$ of codimension $k \geq 1$. We use here the same notation as in Section 3 . Before stating the lemma below, let us recall the definition of the projection $\pi_{r}: \Xi_{2} \backslash M \rightarrow \partial \Xi_{r}:$

$$
\pi_{r}(x):=\pi(x)+r \frac{x-\pi(x)}{d(x)} .
$$

Note that, if $0<r, \lambda \leq 2$, then $\left.\pi_{r}\right|_{\partial \Xi_{\lambda}}: \partial \Xi_{\lambda} \rightarrow \partial \Xi_{r}$ is a smooth diffeomorphism between the manifolds $\partial \Xi_{\lambda}$ and $\partial \Xi_{r}$.

Throughout the Appendix, we will use the notation

$$
\Theta(x, r):=\frac{1}{r^{k-1}} J\left(\left.\pi_{r}\right|_{\partial \Xi_{\lambda}}\right), \quad x \in \partial \Xi_{\lambda}, \quad r, \lambda \in(0,2],
$$

where $J\left(\left.\pi_{r}\right|_{\partial \Xi_{\lambda}}\right)$ denotes the Jacobian of the map $\left.\pi_{r}\right|_{\partial \Xi_{\lambda}}$, so that

$$
\int_{\partial \Xi_{r}} v=\int_{\partial \Xi_{\lambda}} v \circ \pi_{r}(\xi) \Theta(\xi, r) r^{k-1} d \sigma_{\lambda}(\xi), \quad \forall v \in L^{1}\left(\partial \Xi_{r}\right)
$$

or equivalently, by the coarea formula,

$$
\int_{\Xi_{r}} v=\int_{0}^{r} \int_{\partial \Xi_{\lambda}} v \circ \pi_{s}(\xi) \Theta(\xi, s) s^{k-1} d \sigma_{\lambda}(\xi) d s, \quad \forall v \in L^{1}\left(\Xi_{r}\right) .
$$

We should remark at this point that the choice of the normalization factor $\frac{1}{r^{k-1}}$ comes from the degeneracy rate of $J\left(\left.\pi_{r}\right|_{\partial \Xi_{\lambda}}\right)$ as $r \downarrow 0$, as we shall see in Lemma 12.

In the next lemma we present some properties of this function, which were used in some of the main results in this paper. We handle only the case of codimension $k \geq 2$. Since we are mostly interested in the limit behavior of $\Theta(\cdot, r)$ as $r \downarrow 0$, we shall consider $\Theta$ as a function defined on $\partial \Xi_{1} \times(0,2]$, i.e., we take $\lambda=1$ in equations (106) and (107).

Lemma 12 Suppose $M \subset \mathbb{R}^{N}$ is a compact manifold, without boundary, of codimension $k \geq 2$. Then $\Theta \in C^{\infty}\left(\partial \Xi_{1} \times[0,2]\right)$ and satisfies:

(i) there exists $a>0$ such that $\Theta \geq a>0$ on $\partial \Xi_{1} \times[0,2]$; 
(ii) there exist smooth functions $\alpha, \beta$ defined on $\partial \Xi_{1}$ such that

$$
\Theta(\xi, r)=\alpha(\xi)+r^{k} \beta(\xi), \quad \forall(\xi, r) \in \partial \Xi_{1} \times[0,2] .
$$

Proof. Instead of computing $J\left(\left.\pi_{s}\right|_{\partial \Xi_{1}}\right)$ directly in (105) to get the desired properties of $\Theta$, we shall try to find another representation for the function $\Theta$. We proceed as follows:

Given a small geodesic neighborhood $U \subset M$, let $h: U \times B_{2}^{k} \rightarrow \pi^{-1}(U) \times$ int $\Xi_{2}$ be a diffeomorphism such that $h\left(z_{1}, 0\right)=z_{1}, \pi\left(h\left(z_{1}, \cdot\right)\right)=z_{1}$, and $h\left(z_{1}, \cdot\right)$ is an affine linear isometry for each $z_{1} \in U$.

Using the parametrization of $\Xi_{r}$ induced by $h$ and the coarea formula, we have

$$
\begin{aligned}
\int_{\Xi_{r} \cap \pi^{-1}(U)} v & =\int_{U} \int_{B_{r}^{k}} v \circ h J h \\
& =\int_{0}^{r} \int_{U} \int_{\partial B_{s}^{k}} v \circ h J h d \sigma_{s} d z_{1} d s \\
& =\int_{0}^{r} \int_{U} \int_{S^{k-1}}^{r} v\left(h\left(z_{1}, s \zeta\right)\right) J h\left(z_{1}, s \zeta\right) s^{k-1} d \sigma(\zeta) d z_{1} d s \\
& =\int_{0}^{r} \int_{U} \int_{S^{k-1}} v \circ h \circ j_{s}\left(z_{1}, \zeta\right) J h \circ j_{s}\left(z_{1}, \zeta\right) s^{k-1} d \sigma(\zeta) d z_{1} d s
\end{aligned}
$$

where $j_{s}\left(z_{1}, \zeta\right):=\left(z_{1}, s \zeta\right)$.

Therefore, we get the following expression for the integral of $v$ on $\Xi_{r} \cap$ $\pi^{-1}(U)$ :

$$
\begin{aligned}
& \int_{\Xi_{r} \cap \pi^{-1}(U)} v \\
& \quad=\int_{0}^{r} \int_{\partial \Xi_{1} \cap \pi^{-1}(U)} v \circ \pi_{s}\left[J h \circ j_{s} \circ h^{-1} J\left(\left.h^{-1}\right|_{\partial \Xi_{1}}\right)\right] s^{k-1} d \sigma d s,
\end{aligned}
$$

where we used the fact that, by our very choice of $h$, we must have $\pi_{s}=$ $h \circ j_{s} \circ h^{-1}$ on $\partial \Xi_{1}$. If we compare the identities (107) and (109), we then conclude that

$$
\Theta=J h \circ j_{r} \circ h^{-1} J\left(\left.h^{-1}\right|_{\partial \Xi_{1}}\right) \quad \text { on }\left(\partial \Xi_{1} \cap \pi^{-1}(U)\right) \times(0,2] .
$$

Since $U$ was an arbitrary small geodesic neighborhood of $M$ and $h$ was a diffeomorphism, (110) immediately implies that $\Theta \in C^{\infty}\left(\partial \Xi_{1} \times[0,2]\right)$ and $\Theta>0$ on $\partial \Xi_{1} \times[0,2]$, so that $(i)$ must hold. 
In order to prove $(i i)$, we first rewrite (110) as

$$
\begin{aligned}
& \Theta(h(z), r)=J h\left(z_{1}, r z_{2}\right) J\left(\left.h^{-1}\right|_{\partial \Xi_{1}}\right)(h(z)), \\
& \forall(z, r) \in\left(U \times S^{k-1}\right) \times[0,2] .
\end{aligned}
$$

By choosing a smaller open subset of $U$ if necessary, we may assume we have a parametrization $p: \mathbb{R}^{N-k} \rightarrow U$. Next, define $\tilde{h}: \mathbb{R}^{N-k} \times B_{2}^{k} \rightarrow \Xi_{2}$ by $\tilde{h}\left(y_{1}, y_{2}\right):=h\left(p\left(y_{1}\right), y_{2}\right)$, so that

$$
J \tilde{h}\left(y_{1}, y_{2}\right)=J h\left(p\left(y_{1}\right), y_{2}\right) J p\left(y_{1}\right) .
$$

In view of (111) and (112), in order to show that $\Theta$ may be written as (108), it suffices to prove the following decomposition for $J \tilde{h}$ :

$$
J \tilde{h}(y)=\tilde{\alpha}\left(y_{1}\right)+\tilde{\beta}(y), \quad \forall y=\left(y_{1}, y_{2}\right) \in \mathbb{R}^{N-k} \times B_{2}^{k},
$$

where $\tilde{\alpha}, \tilde{\beta}$ are smooth and $\tilde{\beta}\left(y_{1}, y_{2}\right)$ is a homogeneous polynomial of order $k$ with respect to the $y_{2}$-variable, for each $y_{1} \in \mathbb{R}^{N-k}$.

From the properties of $h$, we may write it more explicitly as

$$
h\left(z_{1}, z_{2}\right)=z_{1}+T\left(z_{1}\right) z_{2}, \quad \forall\left(z_{1}, z_{2}\right) \in U \times B_{2}^{k},
$$

for some linear isometry $T\left(z_{1}\right): \mathbb{R}^{k} \rightarrow \mathbb{R}^{N-k}, z_{1} \in U$, so that

$$
\tilde{h}\left(y_{1}, y_{2}\right)=p\left(y_{1}\right)+T\left(p\left(y_{1}\right)\right) y_{2}=: p\left(y_{1}\right)+\tilde{T}\left(y_{1}\right) y_{2},
$$

which implies

$$
\begin{aligned}
J \tilde{h}\left(y_{1}, y_{2}\right) & =\operatorname{det}\left(D p\left(y_{1}\right)+D \tilde{T}\left(y_{1}\right) y_{2}, \tilde{T}\left(y_{1}\right)\right) \\
& =\operatorname{det}\left(D p\left(y_{1}\right), \tilde{T}\left(y_{1}\right)\right)+\operatorname{det}\left(D \tilde{T}\left(y_{1}\right) y_{2}, \tilde{T}\left(y_{1}\right)\right) .
\end{aligned}
$$

Now (113) follows if we take

$$
\begin{gathered}
\tilde{\alpha}\left(y_{1}\right):=\operatorname{det}\left(D p\left(y_{1}\right), \tilde{T}\left(y_{1}\right)\right), \\
\tilde{\beta}\left(y_{1}, y_{2}\right):=\operatorname{det}\left(D \tilde{T}\left(y_{1}\right) y_{2}, \tilde{T}\left(y_{1}\right)\right) .
\end{gathered}
$$

In particular, note that $\tilde{\beta}\left(y_{1}, \cdot\right)$ is a homogeneous polynomial of order $k$. As we already remarked before, (111), (112) and (113) imply (ii). This concludes the proof of the lemma.

The following corollary gives some estimates we needed in the proof of Theorem 8: 
Corollary 13 For any $j \geq 0$ and $x \in \Xi_{1} \backslash M$,

$$
D_{x}^{j}\left[\frac{\partial^{i} \Theta}{\partial r^{i}}\left(\pi_{1}(x), d(x)\right)\right]=\left\{\begin{array}{ll}
O\left(d^{k-i-j}\right) & \text { if } 1 \leq i \leq k \\
0 & \text { if } i>k
\end{array} .\right.
$$

In particular, estimates (86) and (87) hold.

Proof. Firstly, we see from (108) that we only need to prove (114) for $1 \leq i \leq k$. If we differentiate (108) with respect to $r$ and evaluate the resulting expression at the point $(\xi, r)=\left(\pi_{1}(x), d(x)\right)$, for some $x \in \Xi_{2} \backslash M$, we get

$$
\frac{\partial^{i} \Theta}{\partial r^{i}}\left(\pi_{1}(x), d(x)\right)=\frac{k !}{(k-i) !} d(x)^{k-i} \beta\left(\pi_{1}(x)\right) .
$$

In particular, (114) with $j=0$ (and any $i \leq k$ ) follows from the expression above.

Next, we assume $j \geq 1$. Instead of differentiating (115) directly with respect to $x$, we shall write it in terms of local coordinates conveniently chosen, as we did in the proof of Lemma 12.

For a sufficiently small geodesic neighborhood $U \subset M$, we can find a parametrization $p: \mathbb{R}^{N-k} \rightarrow U$ and a diffeomorphism $h: U \times B_{2}^{k} \rightarrow$ $\pi^{-1}(U) \cap$ int $\Xi_{2}$ such that $h\left(z_{1}, 0\right)=z_{1}, \pi\left(h\left(z_{1}, \cdot\right)\right)=z_{1}$, and $h\left(z_{1}, \cdot\right)$ is an affine linear isometry for each $z_{1} \in U$.

Define $\tilde{h}(y):=h\left(p\left(y_{1}\right), y_{2}\right), y \in B_{10}^{N-k} \times B_{2}^{k}$, so that $\tilde{h}$ is a diffeomorphism between $B_{10}^{N-k} \times B_{2}^{k}$ and $\pi^{-1}\left(p\left(B_{10}^{N-k}\right)\right) \cap$ int $\Xi_{2}=: \mathcal{V}$; moreover, the derivatives of $\tilde{h}$ and $\tilde{h}^{-1}$ are bounded (which explains why we defined $\tilde{h}$ using $B_{10}^{N-k}$, instead of $\mathbb{R}^{N-k}$ ).

Given $x \in \mathcal{V} \backslash M$, let $y \in B_{10}^{N-k} \times B_{2}^{k} \backslash\{0\}$ be such that $\tilde{h}(y)=x$. Using the properties of $\tilde{h}$ (or rather of $h$ ), we may write (115) as

$$
\begin{aligned}
\frac{\partial^{i} \Theta}{\partial r^{i}}\left(\pi_{1}(x), d(x)\right) & =\frac{k !}{(k-i) !}\left|y_{2}\right|^{k-i} \beta\left(\pi_{1}(\tilde{h}(y))\right) \\
& =\frac{k !}{(k-i) !}\left|y_{2}\right|^{k-i} \beta\left(\tilde{h}\left(y_{1}, \frac{y_{2}}{\left|y_{2}\right|}\right)\right) \\
& =: \frac{k !}{(k-i) !}\left|y_{2}\right|^{k-i} \tilde{\beta}\left(y_{1}, \frac{y_{2}}{\left|y_{2}\right|}\right)=: F_{i}(y) .
\end{aligned}
$$

One can now check that the derivatives of $F$ satisfy

$$
\left|D^{j} F_{i}(y)\right| \leq C_{i j}\left|y_{2}\right|^{k-i-j}, \quad \forall y \in B_{10}^{N-k} \times B_{2}^{k} \backslash\{0\}, \quad \forall j \geq 1 .
$$


If we now apply the chain rule to (116), then the estimates above and the boundedness of the derivatives of $\tilde{h}^{-1}$ will imply that (114) holds for $j \geq 1$.

Finally, estimates are readily checked using (114) and the fact that $\Theta \geq a>$ 0 on $\partial \Xi_{1} \times[0,1]$.

Acknowledgments: We are indebted to Y. Li for pointing out the geometric interpretation to Kato's inequality which led us to our results on singularities for Laplace's equation we present in this paper. We would also like to thank H. Brezis for the extremely interesting discussions, for mentioning improvements in our results and for his encouragement. Finally, we are grateful to A. Ancona who brought to our attention the connection between Theorem 4 and a classical result in Potential Theory.

\section{References}

[BaPi] P. Baras and M. Pierre, Singularités éliminables pour des équations semi-linéaires, Ann. Inst. Fourier (Grenoble) 34 (1984), 185-206.

[BrL] H. Brezis and P.L. Lions, A note on isolated singularities for linear elliptic equations, Mathematical analysis and applications, Part A, Adv. in Math. Suppl. Stud., 7a, Academic Press, New YorkLondon, 1981, 263-266.

[BeBrC] P. Bénilan, H. Brezis and M.G. Crandall, A semilinear equation in $L^{1}\left(\mathbb{R}^{N}\right)$, Ann. Scuola Norm. Sup. Pisa Cl. Sci. (4) 2 (1975). $523-555$.

[EG] L.C. Evans and R.F. Gariepy, Measure theory and fine properties of functions, Boca Raton, FL, CRC Press, 1992.

[H] L.L. Helms, Introduction to potential theory, New York, Wiley \& Sons, 1969. (Pure and Applied Mathematics, Vol. XXII)

[K] T. Kato, Schrödinger operators with singular potentials, Israel J. Math. 13 (1972), 135-148.

[MPa] R. Mazzeo and F. Pacard, A construction of singular solutions for a semilinear elliptic equation using asymptotic analysis, J. Differential Geom. 44 (1996), 331-370.

[S] L. Schwartz, Théorie des distributions. Tomes I et II., Paris, Hermann \& Cie., 1950 and 1951. 
[V1] L. Véron, Singularités éliminables d'équations elliptiques non linéaires. J. Differential Equations 41 (1981), 87-95.

[V2] L. Véron, Singularities of solutions of second order quasilinear equations, Harlow, Longman, 1996. (Pitman Research Notes in Mathematics Series 353). 